\title{
SBS 0335-052E+W: deep VLT/FORS+UVES spectroscopy of the pair of the lowest-metallicity blue compact dwarf galaxies ${ }^{\star \star \star}$
}

\author{
Y. I. Izotov ${ }^{1,2}$, N. G. Guseva ${ }^{1,2}$, K. J. Fricke ${ }^{3,2}$, and P. Papaderos ${ }^{4,5}$ \\ 1 Main Astronomical Observatory, Ukrainian National Academy of Sciences, Zabolotnoho 27, Kyiv 03680, Ukraine \\ e-mail: izotov@mao.kiev.ua \\ 2 Max-Planck-Institute for Radioastronomy, Auf dem Hügel 69, 53121 Bonn, Germany \\ 3 Institute for Astrophysics, University of Göttingen, Friedrich-Hund-Platz 1, 37077 Göttingen, Germany \\ ${ }^{4}$ Instituto de Astrofísica de Andalucía (CSIC), Camino Bajo de Huétor 50, Granada 18008, Spain \\ 5 Department of Astronomy and Space Physics, Uppsala University, Box 515, 75120 Uppsala, Sweden
}

Received 27 February 2009 / Accepted 25 May 2009

\section{ABSTRACT}

Context. We present deep archival VLT/FORS1+UVES spectroscopic observations of the system of two blue compact dwarf (BCD) galaxies SBS 0335-052E and SBS 0335-052W.

Aims. Our aim is to derive element abundances in different $\mathrm{H}$ II regions of this unique system of galaxies and to study spatial abundance variations.

Methods. The electron temperature $T_{\mathrm{e}}$ (O III) in all $\mathrm{H}$ II regions, except for one, is derived from the [O III] $\lambda 4363 /(\lambda 4959+\lambda 5007)$ flux ratio. We determine ionic abundances of helium, nitrogen, oxygen, neon, sulfur, chlorine, argon and iron. The empirical relations for ionization correction factors are used to derive total abundances of these elements.

Results. The oxygen abundance in the brighter eastern galaxy varies in the range 7.11 to 7.32 in different $\mathrm{H}$ II regions supporting previous findings and suggesting the presence of oxygen abundance variations on spatial scales of $\sim 1-2 \mathrm{kpc}$. Good seeing during FORS observations allowed us to extract spectra of four H II regions in SBS 0335-052W. The oxygen abundance in the brightest region No. 1 of SBS $0335-052 \mathrm{~W}$ is $7.22 \pm 0.07$, consistent with previous determinations. Three other $\mathrm{H}$ II regions are much more metal-poor with an unprecedently low oxygen abundance of $12+\log \mathrm{O} / \mathrm{H}=7.01 \pm 0.07$ (region No. 2), $6.98 \pm 0.06$ (region No. 3), and $6.86 \pm 0.14$ (region No. 4). These are the lowest oxygen abundances ever derived in emission-line galaxies, supporting earlier conclusions that SBS $0335-052 \mathrm{~W}$ is the lowest-metallicity emission-line galaxy known. Helium abundances derived for the brightest $\mathrm{H}$ II regions of both galaxies are mutually consistent. We derive weighted mean He mass fractions of $0.2485 \pm 0.0012$ and $0.2514 \pm$ 0.0012 for two different sets of He I emissivities. The ratios of neon and sulfur to oxygen abundance are similar to the respective ratios obtained for other emission-line galaxies. On the other hand, the chlorine-to-oxygen abundance ratio in SBS 0335-052E is lower, while the argon-to-oxygen abundance ratio is higher than those in other low-metallicity galaxies. The Fe/O abundance ratios in different regions of SBS 0335-052E are among the highest for emission-line galaxies implying that iron is almost entirely not depleted onto dust grains despite dust being detected in this galaxy in earlier ISO and Spitzer observations. The N/O abundance ratio in both galaxies is slightly higher than that derived for other BCDs with $12+\log \mathrm{O} / \mathrm{H}<7.6$. This implies that the N/O in extremely metal-deficient galaxies could increase with decreasing metallicity.

Key words. galaxies: fundamental parameters - galaxies: starburst - galaxies: abundances

\section{Introduction}

The pair of extremely metal-deficient blue compact dwarf (BCD) galaxies SBS 0335-052E and SBS 0335-052W plays a key role in understanding physical conditions in the interstellar medium, star formation, and stellar evolution at very low metallicity. The first spectroscopic observations and abundance determinations of the brighter eastern galaxy were completed $\sim 20$ years ago (Izotov et al. 1990a,b,c). The fainter western galaxy was discovered and spectroscopically studied first by Pustilnik et al. (1997). Based on the VLA H I mapping

\footnotetext{
* Based on observations collected at the European Southern Observatory, Chile, ESO program 69.C-0203(A), 71.B-0055(A)), 70.B-0717(A) and 68.B-0310(A).

$\star \star$ Tables $1-8$ are only available in electronic form at

http://www. aanda.org
}

Pustilnik et al. (2001) showed that both galaxies are embedded in a large $\mathrm{H}$ I cloud (66 by $22 \mathrm{kpc}$ ) and constitute a pair (separated by $22 \mathrm{kpc}$ ) of physically related galaxies.

First abundance determinations of the brightest knot of SBS 0335-052E inferred a very low oxygen abundance in the range $12+\log \mathrm{O} / \mathrm{H}=7.0-7.3$ (Izotov et al. 1990a,b,c; Melnick et al. 1992), implying that it is one of the most metaldeficient BCD known. Later spectroscopic studies of SBS 0335052E indicated that the oxygen abundances of the brightest regions are $12+\log \mathrm{O} / \mathrm{H} \sim 7.3$ (Izotov et al. 1997b, 2006a; Papaderos et al. 2006). They also detected variations in the oxygen abundance in the range of $12+\log \mathrm{O} / \mathrm{H}=7.1-7.3$ on spatial scales of $\sim 1-2 \mathrm{kpc}$. On the other hand, the oxygen abundance in SBS $0335-052 \mathrm{~W}$ is lower yet, $12+\log \mathrm{O} / \mathrm{H}=$ $7.12 \pm 0.03$ (Izotov et al. 2005), making it the lowest-metallicity emission-line galaxy known to date. For comparison, the oxygen 

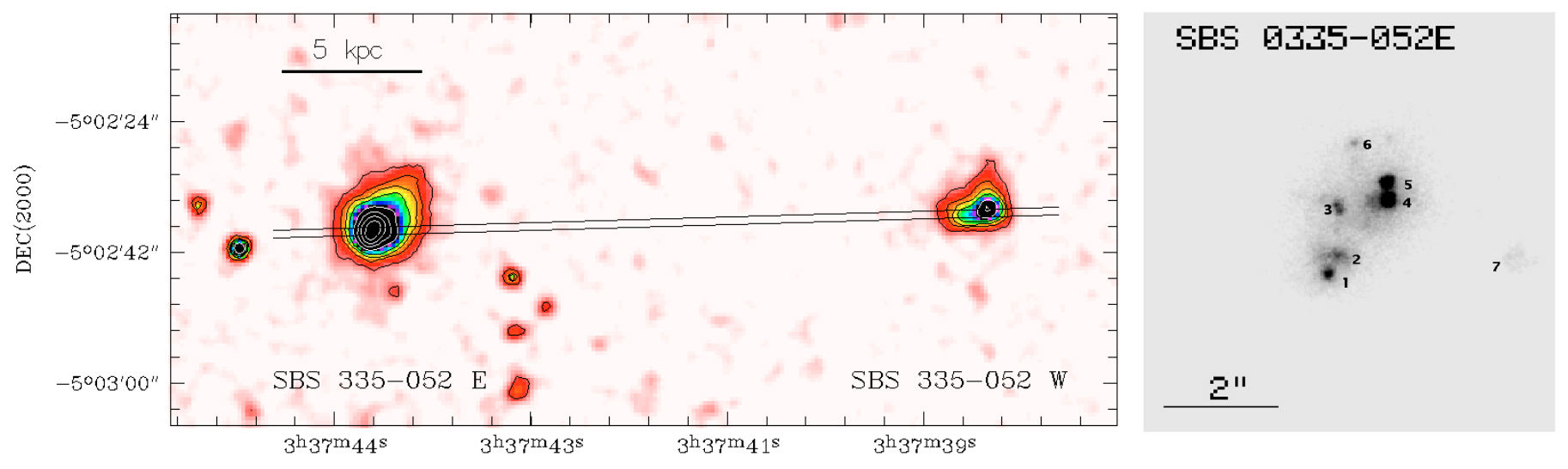

Fig. 1. Left: $2.2 \mathrm{~m}$ Calar Alto telescope B image of the galaxy system SBS 0335-052E and SBS 0335-052W. The two straight lines indicate the location of the slit during VLT/FORS observations. Right: Archival HST UV image of SBS 0335-052E. The clusters are labelled according to Thuan et al. (1997) and Papaderos et al. (1998).

abundances in the second and third most metal-deficient galaxies DDO 68 (三SDSS J0956+2849) and I Zw 18 are respectively, $7.14 \pm 0.03$ (Izotov \& Thuan 2007) and $7.17 \pm 0.01$ (Thuan \& Izotov 2005).

The extremely low oxygen abundances in the galaxy pair SBS 0335-052E and SBS 0335-052W imply that they are among the most suitable relatively nearby objects (at a distance of $54 \mathrm{Mpc}$ ) for studying the properties of low-metallicity stars and the interstellar medium and interpreting the physical conditions in high-redshift young galaxies. The brighter BCD SBS 0335-052E has been extensively studied in different wavelength ranges. The most imortant results from these studies are the following: 1) star formation in this galaxy resides in several very compact and bright super-star clusters (SSCs) (Thuan et al. 1997; Papaderos et al. 1998; Thompson et al. 2006, 2008). These SSCs are labelled in Fig. 1 (right panel) following Thuan et al. (1997) and Papaderos et al. (1998). 2) Thuan \& Izotov (1997) discovered evidence of stellar winds in the massive stars of SBS 0335-052E based on the HST UV spectroscopic observations of the brightest SSCs. 3) Vanzi et al. (2000) first detected near-infrared emission lines of molecular hydrogen, implying that star formation in SBS 0335-052E occurs in molecular clouds. Thompson et al. $(2006,2008)$ identified this emission with SSCs Nos. 1 and 2. The discovery of $\mathrm{H}_{2}$ emission is in line with the detection of warm and hot dust emission (Thuan et al. 1999; Vanzi et al. 2000; Hunt et al. 2001; Houck et al. 2004) that is also associated with dense star-forming regions. 4) High-ionization emission lines are present in SBS 0335-052E (in particular, [Ne V] 23346,3426 ) indicating that the hard ionizing radiation with photon energies above 7 Ryd is intense in this galaxy. This radiation is most likely to be associated with fast radiative shocks propagating in the dense interstellar medium (Izotov et al. 2001; Thuan \& Izotov 2005).

SBS 0335-052W has not been studied in such detail mainly because it is much fainter than SBS 0335-052E. No HST images have yet been obtained for this galaxy precluding detailed analysis of the morphology of its central region. Ground-based photometric and spectroscopic observations suggest that star formation in SBS 0335-052W is confined to several young clusters, which are not as bright as SSCs in SBS 0335-052E (Pustilnik et al. 1997; Papaderos et al. 1998; Lipovetsky et al. 1999; Izotov et al. 2005; Papaderos et al. 2006). On the other hand, the western galaxy is brighter in the X-ray range (Thuan et al. 2004) and in the H I $\lambda 21 \mathrm{~cm}$ emission line (Pustilnik et al. 2001).
Despite extensive optical spectroscopic studies of both galaxies, additional high-quality spectroscopic observations with large telescopes had been required. First, improving the heavy element abundance determinations is important to the study of abundance variations in extremely low-metallicity environments and understanding the mixing processes in the interstellar medium. Secondly, this system of galaxies is one of the most suitable targets for the determination of the primordial He abundance (Izotov et al. 2007a). To address these problems, we use $8.2 \mathrm{~m}$ Very Large Telescope (VLT) spectroscopic observations of both galaxies obtained with the spectrographs FORS1 and UVES some of these data are of the highest signal-to-noise ratio ( $S / N \gtrsim 100$ in the continuum), spatial resolution $\left(<1^{\prime \prime}\right)$, and spectral resolution $(\sim 0.02-3 \AA)$ ever obtained for these galaxies. The raw data of these observations were extracted from the European Southern Observatory (ESO) archive.

The objective of this paper is to determine with the highest precision, the element abundances in the different regions of SBS 0335-052E and SBS 0335-052W (Fig. 1), to study heavy element abundance variations and derive He abundances.

In Sect. 2, we describe observations and data reduction, and in Sect. 3 we present our results. Our conclusions are presented in Sect. 4.

\section{Observations and data reduction}

\subsection{FORS observations}

The FORS spectra of SBS 0335-052E and SBS 0335-052W were obtained on 10 September, 2002 with the FORS1 spectrograph mounted at the UT3 of the $8.2 \mathrm{~m}$ ESO VLT (ESO program 69.C-0203(A)). The observing conditions were photometric throughout the night.

Two sets of spectra were obtained. Low-resolution spectra were obtained with a grism $300 \mathrm{~V}(\lambda \lambda \sim 3850-7500)$ and a blocking filter GG 375. The grisms 600B $(\lambda \lambda \sim 3560-5970)$ and 600R $(\lambda \lambda \sim 5330-7480)$ for the blue and red wavelength ranges were used in the high-resolution observations. To avoid second-order contamination, the red part of the spectrum was obtained with the blocking filter GG 435 .

The long $\left(\sim 418^{\prime \prime}\right)$ slit with a width of 0.51 was centered on regions Nos. 1 and 2 in SBS 0335-052E and oriented in a direction with position angle of -91.7 , simultaneously crossing the brightest H II region in SBS 0335-052W (Fig. 1, left). 

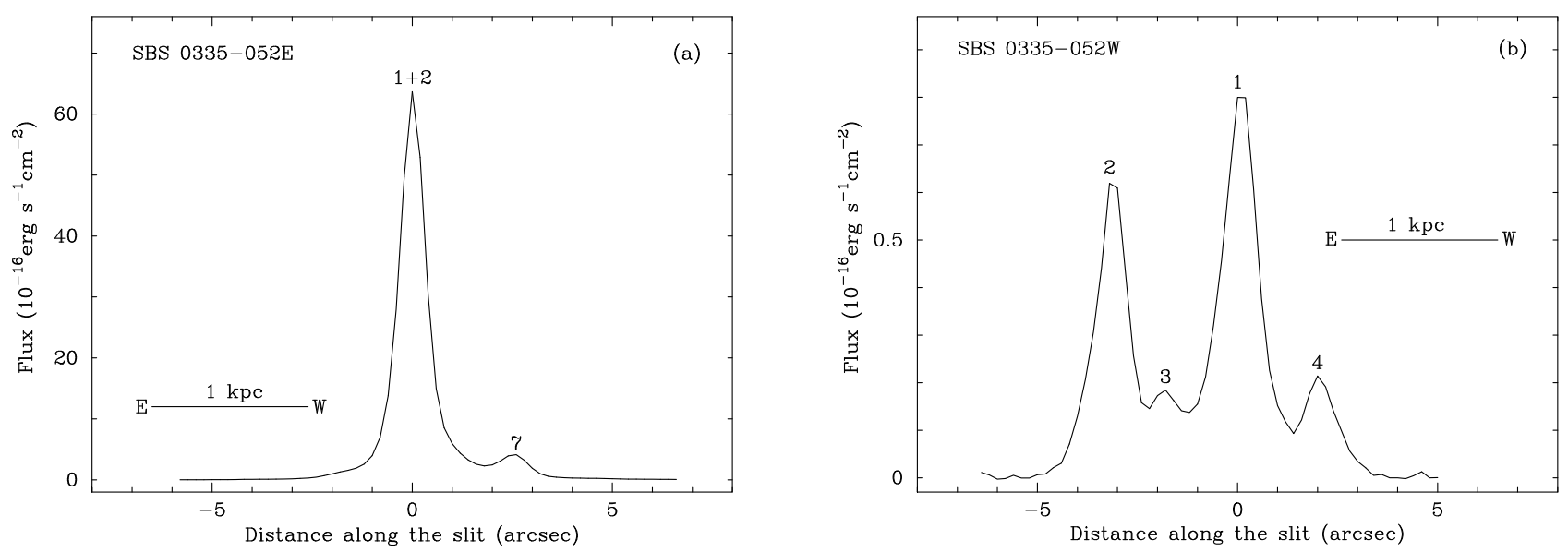

Fig. 2. $\mathrm{H} \beta$ flux distribution along the slit (high-resolution FORS observations). The orientation of the slit is shown in Fig. 1, left. The clusters Nos. $1+2$ and No. 7 in SBS 0335-052E are labelled in a). The clusters Nos. 1 to 4 in SBS 0335-052W are presented in b).

The spatial scale along the slit was 0.2 pixel $^{-1}$ and the resolving power $\lambda / \Delta \lambda=300$ in the low-resolution mode and $\lambda / \Delta \lambda=780$ and 1160 in a high resolution mode for the $600 \mathrm{~B}$ and 600R grisms, respectively. The spectra were obtained at low airmass $\$ 1.1$, so no correction for atmospheric refraction was necessary. The seeing was $\sim 0{ }^{\prime} 9$ during the low-resolution observations, 0.'62-0,'77 during the high-resolution observations in the blue range, and 1.'00-1'.07 during the high-resolution observations in the red range. The total integration time for the low-resolution observations was $720 \mathrm{~s}(6 \times 120 \mathrm{~s})$. The longer exposures were taken for the high-resolution observations and consisted of $4320 \mathrm{~s}(6 \times 720 \mathrm{~s})$ and $2773 \mathrm{~s}(4 \times 600 \mathrm{~s}$ and $1 \times$ 373 s) for the blue and red parts, respectively. In Fig. 2, we show the distribution of the $\mathrm{H} \beta$ emission line flux along the slit. The bright regions Nos. $1+2$ and the much fainter region No. 7 in SBS 0335-052E are labelled in Fig. 2a. The regions No. 1 through No. 4 in SBS 0335-052W are denoted in Fig. 2b according to their brightness in the continuum. We note, however, that the $\mathrm{H} \beta$ line of the region No. 4 is slightly stronger than that of the region No. 3. We note also, that, despite the small angular separation between regions Nos. 2 and 3 and between Nos. 1 and 4 , the good photometric conditions during observations and the small CCD pixel size allowed us to resolve all four $\mathrm{H}$ II regions in SBS 0335-052W and extract one-dimensional spectra for all of them.

\subsection{UVES observations}

Spectra of regions No. $1+2$ of SBS 0335-052E were also obtained on 6 September 2003 with the UVES echelle spectrograph mounted at the VLT (UT2) ESO telescope (ESO program 71.B-0055(A)). The gratings $\mathrm{CD} \# 2$ with the central wavelengths $3900 \AA$ and $4370 \AA$ in the blue arm, CD\#3 with the central wavelength $6000 \AA$ and filter BK7-5, and CD\#4 with the central wavelength $9000 \AA$ and filter OG590 in the red arm were used to provide spectra for the wavelength range $\lambda \lambda 3300-10000$ over 124 orders. The slits with length of $8^{\prime \prime}$ and $12^{\prime \prime}$ for blue and red parts of the spectra, respectively, and width $3^{\prime \prime}$ were centered on regions Nos. $1+2$. The spatial scale along the slit was 0 '.246 and $0^{\prime} 182$ for the blue and red arms, respectively. The galaxy was observed at a low average airmass of 1.13 , so no correction for atmospheric refraction was necessary. The seeing was $0{ }^{\prime} 61-0{ }^{\prime} 63$ at the start and at the end of observation. The spectra were obtained at position angle of $0^{\circ}$ with an exposure time of $3000 \mathrm{~s}$ for the gratings centered on $3900 \AA$ and $6000 \AA$ and of $6300 \mathrm{~s}$ for the gratings centered on $4370 \AA$ and $9000 \AA$.

Two observations were obtained with UVES on 9 November 2002 (ESO program 70.B-0717(A)). The first observation was for the brightest part of SBS 0335-052W with an exposure time of $17550 \mathrm{~s}$ at position angle of $110^{\circ}$ and the second one simultaneously for regions Nos. $1+2$ and Nos. $4+5$ of SBS $0335-052 E$ with an exposure time of $2400 \mathrm{~s}$ at position angle of $150^{\circ}$. The grating CD\#2 centered on $3900 \AA$ and filter HER-5 and grating CD\#3 centered on $5800 \AA$ and filter SHP700 were used resulting in the wavelength range $\lambda \lambda 3250-6950$ over 77 orders for regions Nos. $4+5$ of SBS 0335-052E and for the brightest region of SBS 0335$052 \mathrm{~W}$ ). The slits with length of $8^{\prime \prime}$ (grating CD\#2) and $12^{\prime \prime}$ (grating CD\#3) and width $1^{\prime \prime}$ were used. The spatial scales along

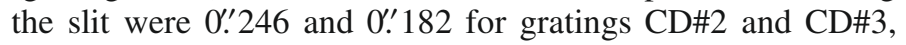
respectively. The spectra were obtained at low average airmasses of 1.17 and 1.08 for SBS 0335-052W and SBS 0335-052E, respectively, so no corrections for atmospheric refraction was required. The seeing was $\sim 0$ !' 8 during the observations of both galaxies.

The UVES observations of the regions Nos. $4+5$ and No. 7 of SBS 0335-052E were obtained on 11 October 2001 (ESO program 68.B-0310(A)). The gratings CD\#1 centered on the wavelength $3460 \AA$ and filter CUS04, and CD\#2 centered on the wavelength $4370 \AA$ and filter CUSO4 were used in the blue arm, while gratings $\mathrm{CD} \# 3$ centered at the wavelength $5800 \AA$ and filter SHP700 and CD\#4 centered at the wavelength $8600 \AA$ and filter OG590 were used in the red arm. This setup resulted in the wavelength range of $\lambda \lambda 3150-9900 \AA$ over 124 orders. The slit length and width were $12^{\prime \prime}$ and 1 ." 5 , respectively. Spatial scales along the slit were 0 '.246 and 0 ' 182 for blue and red arm observations, respectively. All spectra were obtained at position angle of $60^{\circ}$ with an exposure time of $1500 \mathrm{~s}$. Averaged airmasses for $\mathrm{CD} \# 1+\mathrm{CD} \# 3$ and $\mathrm{CD} \# 2+\mathrm{CD} \# 4$ observations were 1.88 and 2.24. Therefore, correction for the atmospheric refraction was required, but it was not applied, presumably because the parallactic angle of $74^{\circ}$ during observations was close to the position angle. This probably has a small effect of atmospheric refraction. The seeing was 0 !'77 and $1^{\prime \prime}, 26$ at the start and the end of observation, respectively. 

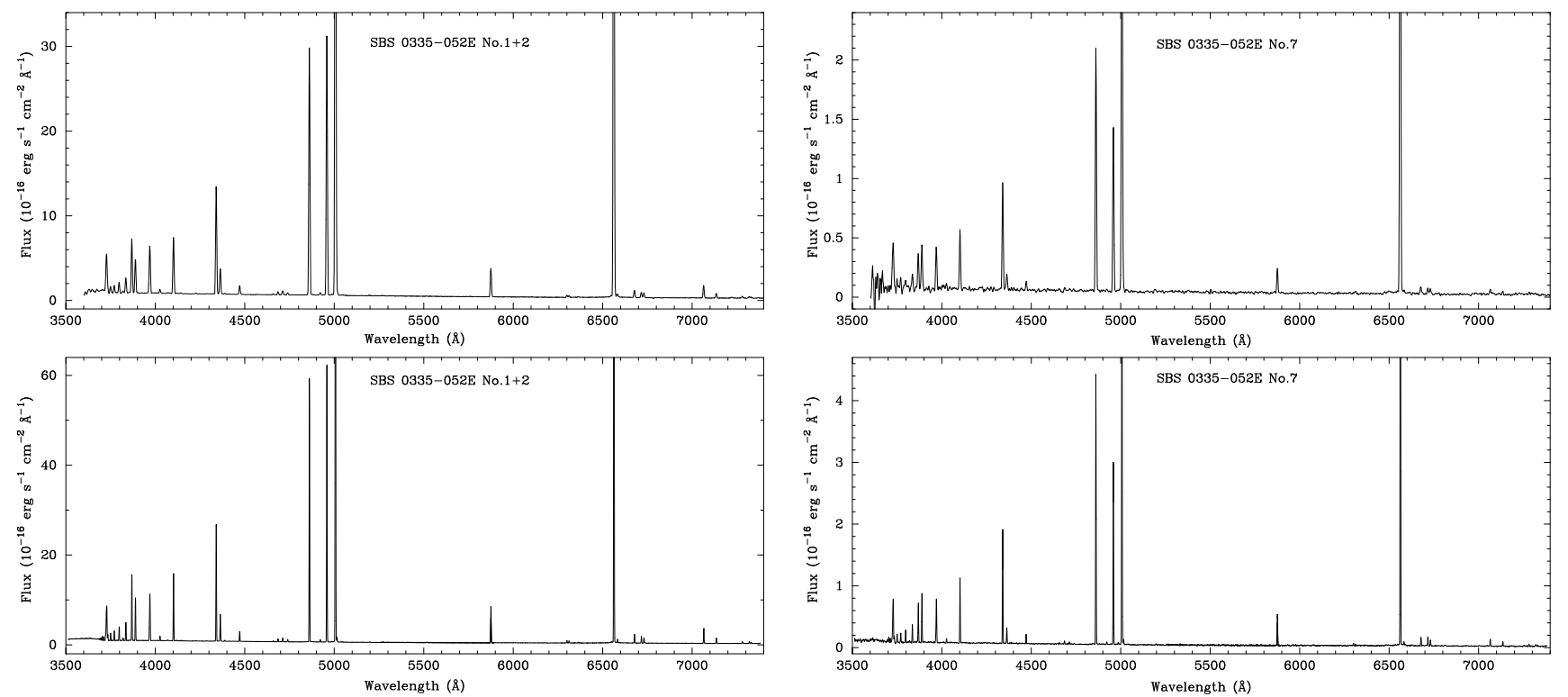

Fig. 3. Flux-calibrated and redshift-corrected FORS low-resolution spectra (upper panel) and FORS high-resolution spectra (lower panel) of regions Nos. $1+2$ (left) and No. 7 (right) in SBS 0335-052E (ESO program 69.C-0203(A)).
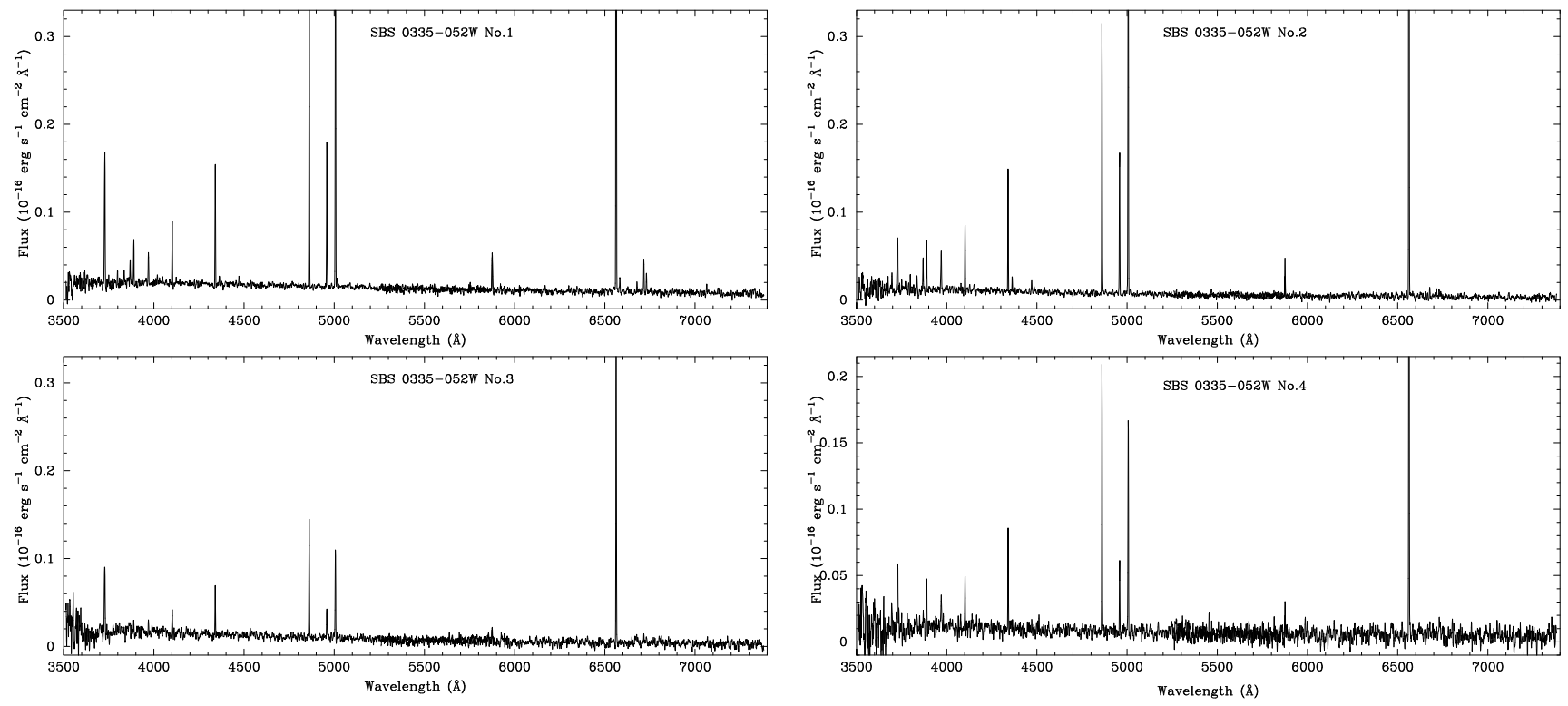

Fig. 4. Flux-calibrated and redshift-corrected FORS high-resolution spectra of regions No. 1 through No. 4 in SBS 0335-052W as labelled in Fig. 2 (ESO program 69.C-0203(A)).

\subsection{Data reduction}

The data were reduced with the IRAF ${ }^{1}$ software package. This included bias-subtraction, flat-field correction, cosmic-ray removal, wavelength calibration, night sky background subtraction, correction for atmospheric extinction, and absolute flux calibration of the two-dimensional spectrum. The spectra were also corrected for interstellar extinction using the reddening curve of Whitford (1958). The flux-calibrated and redshift-corrected onedimensional FORS spectra of the regions Nos. $1+2$ and No. 7 in SBS 0335-052E and four regions in SBS 0335-052W are shown in Figs. 3 and 4, respectively. In Fig. 5, we show the expanded

${ }^{1}$ IRAF is the Image Reduction and Analysis Facility distributed by the National Optical Astronomy Observatory, which is operated by the Association of Universities for Research in Astronomy (AURA) under cooperative agreement with the National Science Foundation (NSF). high-resolution spectrum of SBS 0335-052E Nos. $1+2$ (the same as in Fig. 3) to be able to identify more clearly the numerous weak permitted and forbidden lines. We note the presence of broad $\mathrm{H} \alpha, \mathrm{H} \beta$, and $\mathrm{H} \gamma$ emission in Fig. 5 with fluxes of $\sim 1-2$ percent of the narrow component fluxes, in agreement with the value for the $\mathrm{H} \beta$ emission line obtained by Izotov et al. (2007b) for the same regions Nos. $1+2$. The flux-calibrated and redshift-corrected UVES spectra of regions Nos. $1+2$, Nos. 4+5, and No. 7 of SBS 0335-052E are shown in Figs. 6-8, respectively, and the spectrum of the bright part of SBS 0335-052W is shown in Fig. 9.

Emission-line fluxes were measured using Gaussian profile fitting. The $1 \sigma$ errors of the line fluxes were calculated from the photon statistics in the non-flux-calibrated spectra. The true errors in the line fluxes are probably higher because we do not take into account uncertainties introduced during observations 


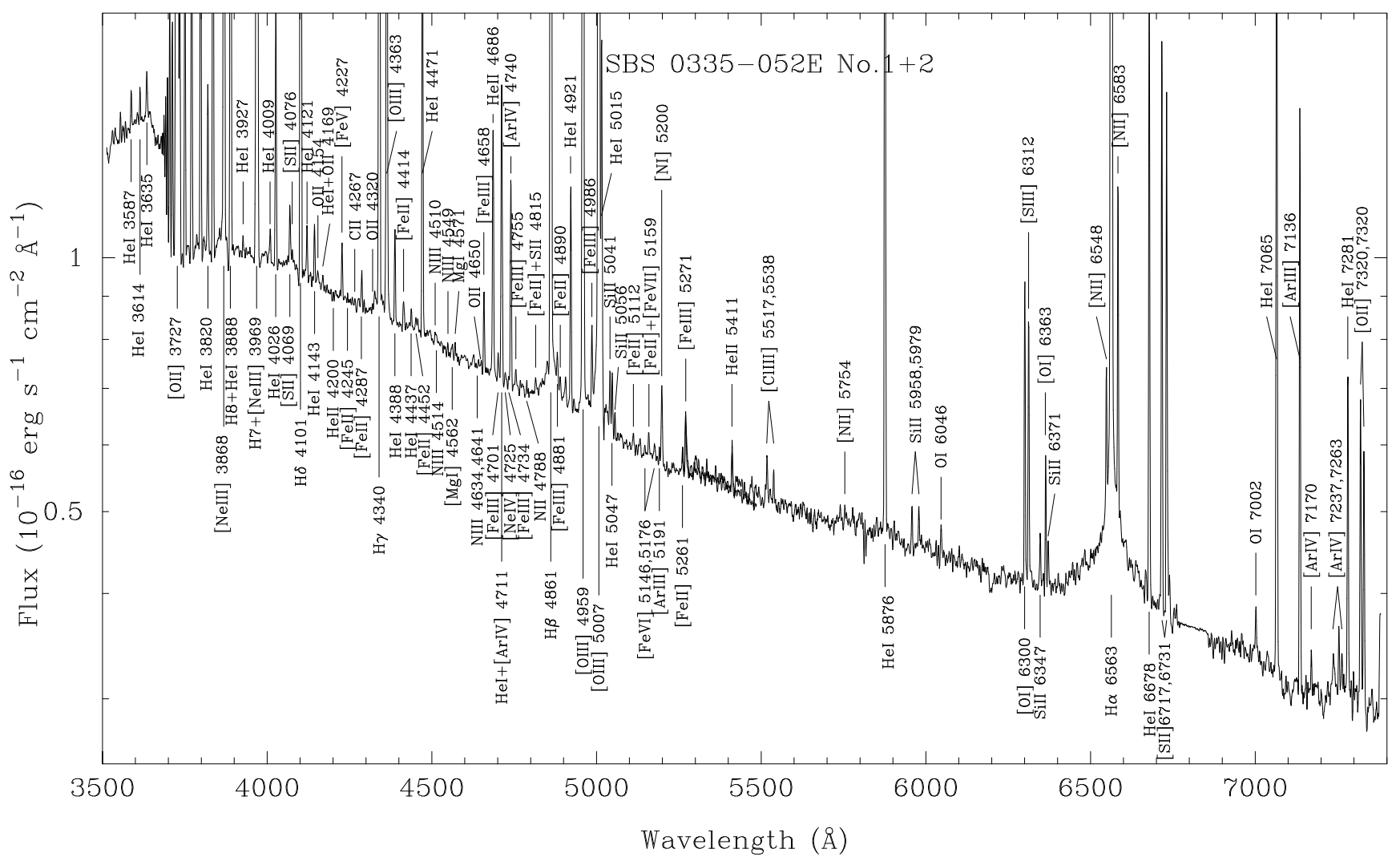

Fig. 5. Flux-calibrated and redshift-corrected FORS high-resolution spectrum of regions Nos. $1+2$ (expanded version of Fig. 3 , left lower panel, to visualise weak emission lines more clearly) (ESO program 69.C-0203(A)). Note the presence of broad emission in the hydrogen lines H $\alpha$, $\mathrm{H} \beta$, and $\mathrm{H} \gamma$. No appreciable broad emission is detected in strong forbidden lines, implying rapid motions of relatively dense ionized gas with an electron number density $N_{\mathrm{e}} \geq 10^{5-6} \mathrm{~cm}^{-3}$.

(e.g., effect of differential refraction) and data reduction. The line flux errors were propagated in the calculations of the elemental abundance errors. The extinction-corrected emission line fluxes $I(\lambda)$ relative to the $\mathrm{H} \beta$ fluxes multiplied by 100 , the extinction coefficients $C(\mathrm{H} \beta)$, the equivalent widths $E W(\mathrm{H} \beta)$, the observed $\mathrm{H} \beta$ fluxes $F(\mathrm{H} \beta)$ and the equivalent widths of the hydrogen absorption lines are listed in Tables 1 and 2 (low-resolution and high-resolution FORS observations), in Table 3 (for weak lines in the high-resolution FORS spectrum of SBS 0335-052E Nos. $1+2$ ), and in Table 4 (UVES observations).

\section{Results}

\subsection{Electron temperature and electron number density}

The electron temperature $T_{\mathrm{e}}$, the ionic and total heavy element abundances were derived following Izotov et al. (2006b). In particular for $\mathrm{O}^{2+}, \mathrm{Ne}^{2+}$ and $\mathrm{Ar}^{3+}$, we adopt the temperature $T_{\mathrm{e}}(\mathrm{O}$ III $)$ directly derived from the [O III] $\lambda 4363 /(\lambda 4959+$ 15007) emission-line ratio. We use $T_{\mathrm{e}}(\mathrm{O}$ II $)$ for the calculation of $\mathrm{O}^{+}, \mathrm{N}^{+}, \mathrm{S}^{+}$, and $\mathrm{Fe}^{2+}$ abundances, and $T_{\mathrm{e}}(\mathrm{S}$ III) for the calculation of $\mathrm{S}^{2+}, \mathrm{Cl}^{2+}$, and $\mathrm{Ar}^{2+}$ abundances.

For all regions in SBS 0335-052E, the electron number densities $N_{\mathrm{e}}(\mathrm{S}$ II) were obtained from the [S II] $\lambda 6717 / \lambda 6731$ emission line ratio, whereas in all regions in SBS 0335-052W, except for region No. 1, the [S II] $\lambda 6717,6731$ emission lines are too weak to allow density determinations. Therefore, for abundance determinations in regions Nos. 2-4 of SBS 0335-052W, we adopt $N_{\mathrm{e}}=10 \mathrm{~cm}^{-3}$. The value of the electron number density makes little difference to the derived abundances since in the low-density limit, which holds for the $\mathrm{H}$ II regions considered here, the element abundances do not depend sensitively on $N_{\mathrm{e}}$. We note that errors of the element abundances do not account for the uncertainties in the ionization correction factors. The electron temperatures $T_{\mathrm{e}}(\mathrm{O}$ III $), T_{\mathrm{e}}(\mathrm{O}$ II $)$, and $T_{\mathrm{e}}(\mathrm{S} \mathrm{III})$, electron number density $N_{\mathrm{e}}(\mathrm{S}$ II $)$, the ionization correction factors $(I C F \mathrm{~s})$, and the ionic and total $\mathrm{N}, \mathrm{O}, \mathrm{Ne}, \mathrm{S}, \mathrm{Cl}, \mathrm{Ar}$, and $\mathrm{Fe}$ abundances derived from the forbidden emission lines are given in Tables 5 and 6 (low-resolution and high-resolution FORS observations), respectively and in Table 7 (UVES observations). We note that the $T_{\mathrm{e}}(\mathrm{S}$ III $)$ derived in all observations lies between $T_{\mathrm{e}}(\mathrm{O}$ III $)$ and $T_{\mathrm{e}}(\mathrm{O} \mathrm{II})$, as expected.

\subsection{Oxygen abundance}

Abundances of $\mathrm{O}^{+}$and $\mathrm{O}^{2+}$ in all regions of SBS 0335-052E and SBS 0335-052W were obtained from the fluxes of forbidden emission lines [O II] $\lambda 3727$ and [O III] $\lambda 4959,5007$. Additionally, for the determination of the $\mathrm{O}^{2+}$ abundance, we used the very weak recombination emission line O II $\lambda 4650$, which was detected in the high-resolution FORS spectrum of regions Nos. $1+2$ in SBS 0335-052E (Table 4). Adopting radiative recombination coefficients from Péquignot et al. (1991), we obtained a $\mathrm{O}^{2+} / \mathrm{H}^{+}$abundance ratio of $(1.50 \pm 0.75) \times 10^{-5}$ from the recombination line flux. This value is consistent with that derived from the forbidden lines (Table 6).

We derived the oxygen abundances of regions $1+2$ in SBS $0335-052 \mathrm{E}$ to be $12+\log \mathrm{O} / \mathrm{H}=7.28 \pm 0.01$ (lowresolution FORS observations), $7.23 \pm 0.01$ (high-resolution FORS observations), and 7.28 \pm 0.01 and $7.27 \pm 0.01$ (for two 


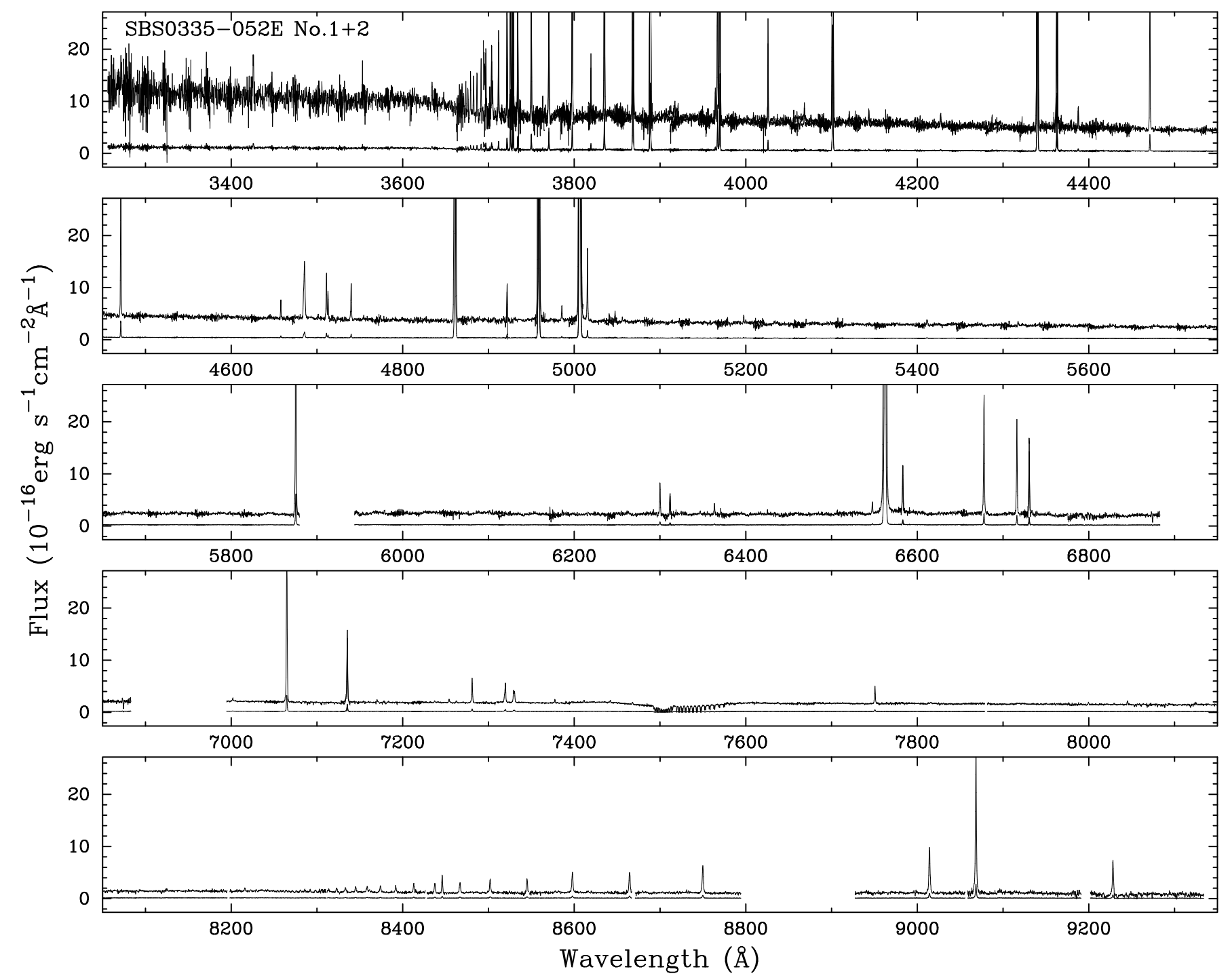

Fig. 6. Flux-calibrated and redshift-corrected UVES spectrum of the regions Nos. $1+2$ in SBS 0335-052E (ESO program 71.B-0055(A)).

different UVES observations). For regions $4+5$, the oxygen abundance was inferred to be $12+\log \mathrm{O} / \mathrm{H}=7.28 \pm 0.01$ and $7.32 \pm 0.01$ for two UVES observations. For cluster 7 , its oxygen abundance is $7.12 \pm 0.04$ (low-resolution FORS observation), $7.15 \pm 0.02$ (high-resolution FORS observation), and $7.24 \pm$ 0.05 (UVES observation). The true differences in the oxygen abundance determinations for the same regions are higher than the calculated errors based on the noise statistics, implying that the errors could be underestimated. In particular, these errors do not include uncertainties introduced by the standard data reduction. Additionally, differences in oxygen abundances could probably be caused by different slit positions and different apertures. The oxygen abundance obtained from a combined spectrum of the brightest regions $1+2+4+5$ is $7.30 \pm 0.01$ and is very close to the value $7.31 \pm 0.01$ obtained by Izotov et al. (1997b) and Thuan \& Izotov (2005) for the brightest part of SBS 0335052E. Our oxygen abundances for regions $4+5$ are close to the value $7.27 \pm 0.02$ obtained by Papaderos et al. (2006). We find that the oxygen abundance in the fainter region 7 is lower than in the brighter regions and compares well with the previous determination of $7.21 \pm 0.02$ by Papaderos et al. (2006). Thus, we confirm the tendency for the oxygen abundance to decrease from the brightest part of SBS 0335-052E to its outer fainter part, suggesting the presence of oxygen abundance variations on spatial scales of $\sim 1-2 \mathrm{kpc}$, in agreement with the finding of Izotov et al. (1997b), Izotov et al. (2006a), and Papaderos et al. (2006).

The oxygen abundance of the whole bright part of SBS 0335-052W obtained from the UVES spectrum is $12+\log \mathrm{O} / \mathrm{H}=7.13 \pm 0.02$. This value is consistent with $12+\log \mathrm{O} / \mathrm{H}=7.12 \pm 0.03$ found by Izotov et al. (2005) from combined $4 \mathrm{~m}$ Kitt Peak, $6.5 \mathrm{~m}$ MMT, and $10 \mathrm{~m}$ Keck II telescope observations, with $12+\log \mathrm{O} / \mathrm{H}=7.11 \pm 0.05$ obtained by Thuan \& Izotov (2005) from MMT observations, and with $12+\log \mathrm{O} / \mathrm{H}=7.13 \pm 0.08$ found by Papaderos et al. (2006) from $3.6 \mathrm{~m}$ ESO telescope observations. Thus, different determinations of the oxygen abundance in the aperture covering the bright part of SBS 0335-052W are in a good agreement.

The determination of the oxygen abundances in individual star-forming regions of SBS $0335-052 \mathrm{~W}$ is a more difficult task because of the faintness of these regions and the small angular separations between them. From $3.5 \mathrm{~m}$ Calar Alto telescope $R, I$ photometry, Lipovetsky et al. (1999) have found that SBS 0335-052W consists at least of three star-forming regions. Given the good seeing during FORS observations, we find 


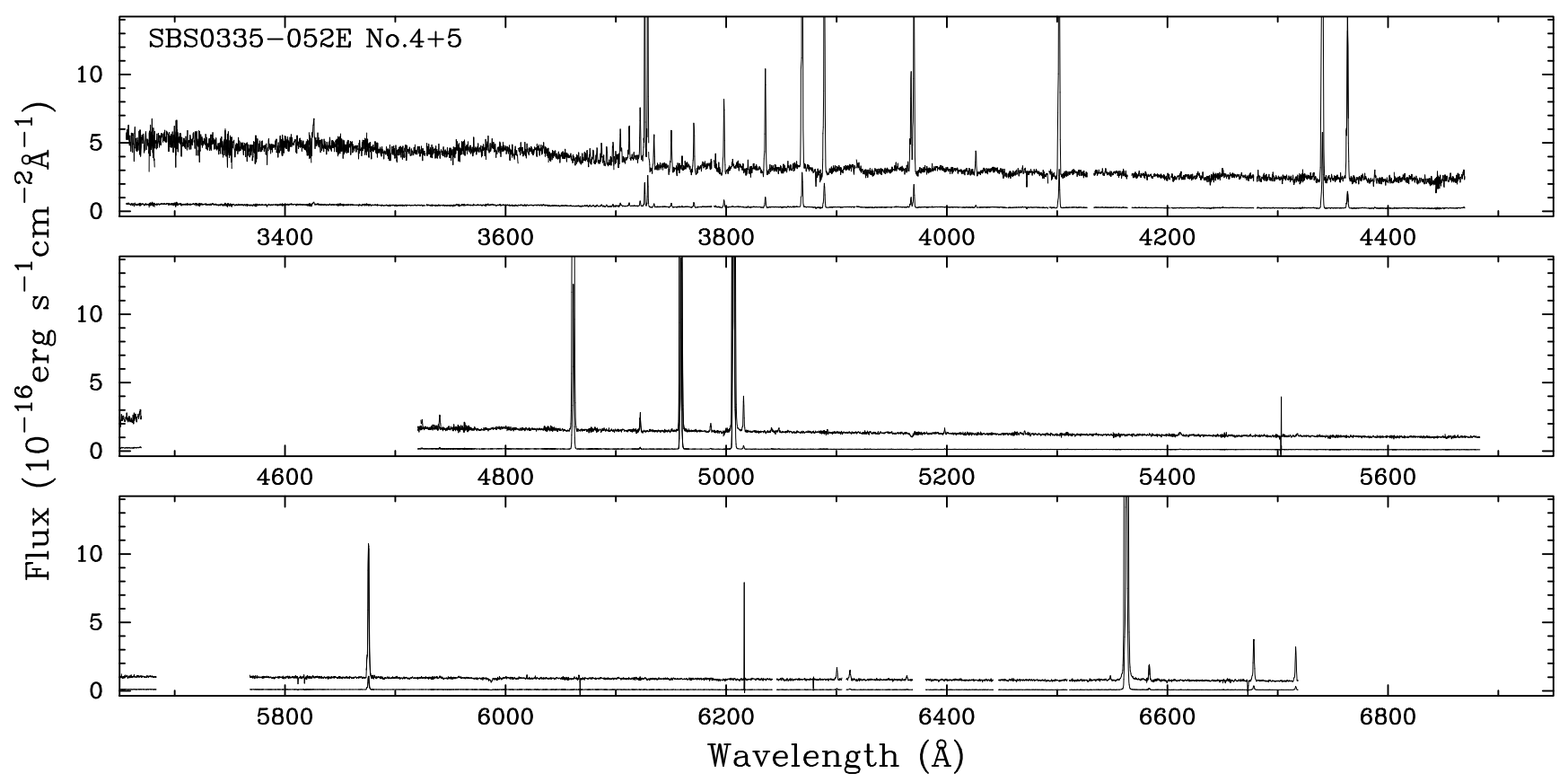

Fig. 7. Flux-calibrated and redshift-corrected UVES spectrum of regions Nos. $4+5$ in SBS 0335-052E (ESO program 70.B-0717(A)).

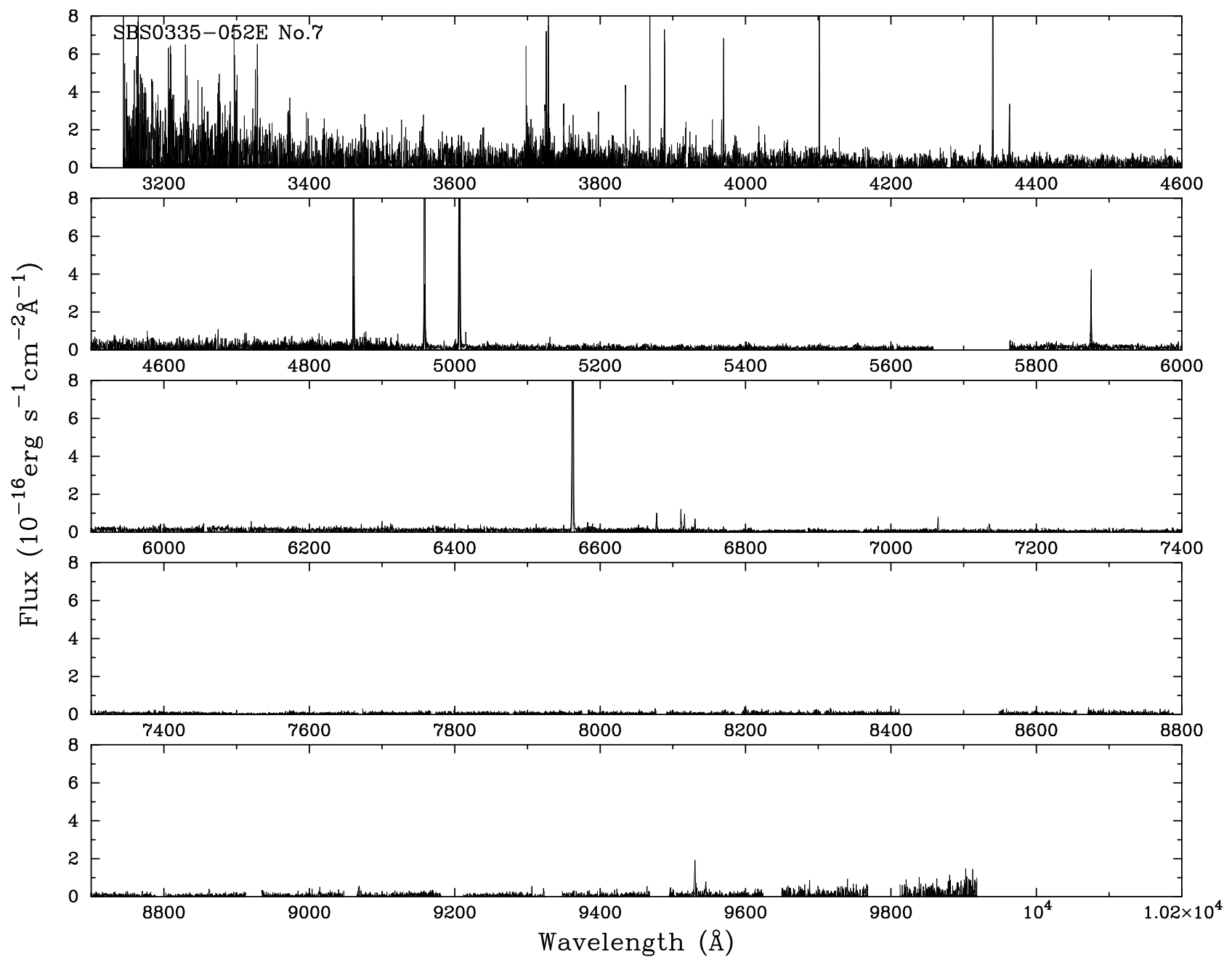

Fig. 8. Flux-calibrated and redshift-corrected UVES spectrum of region No. 7 in SBS 0335-052E (ESO program 68.B-0310(A)). 


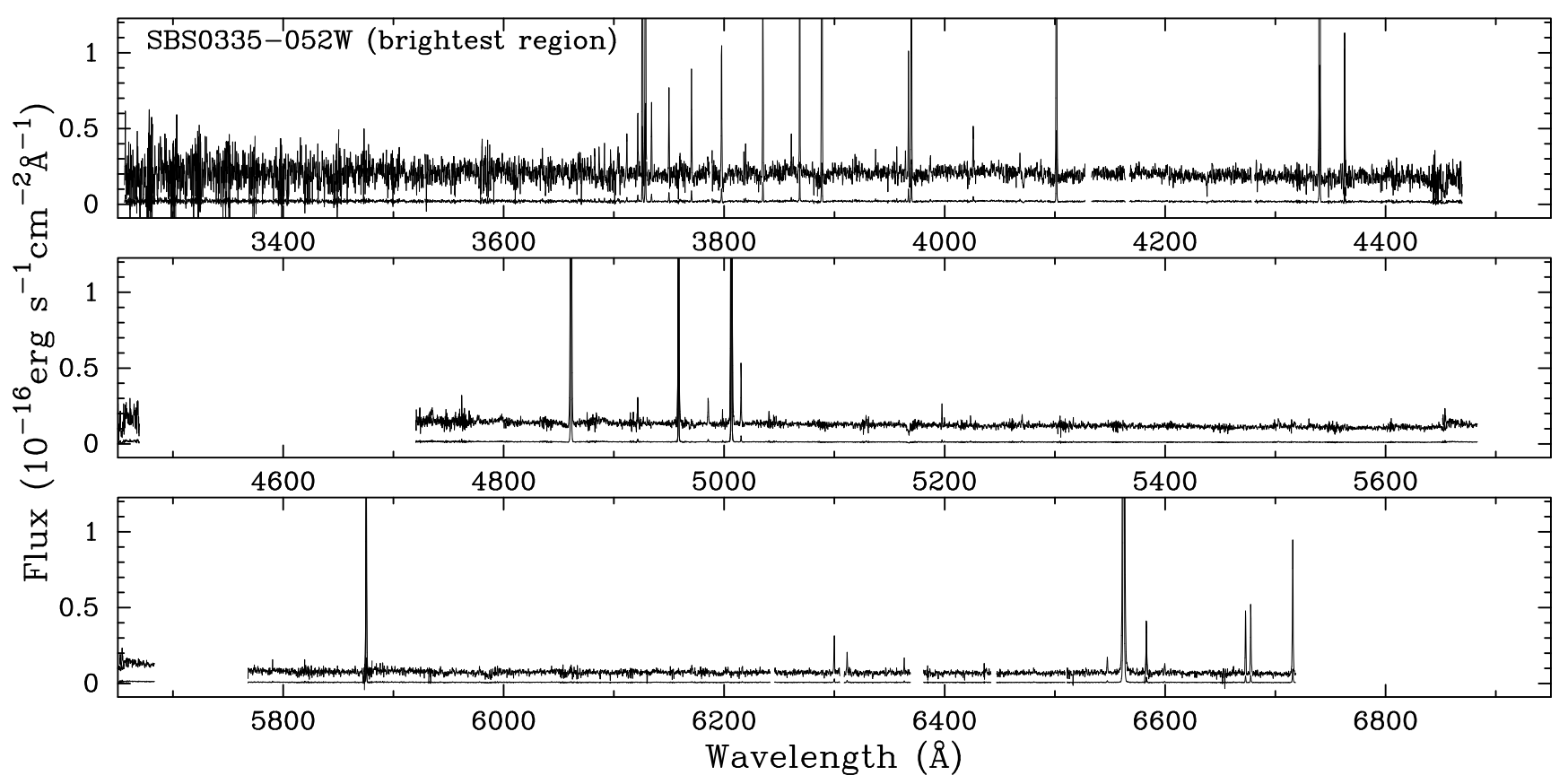

Fig. 9. Flux-calibrated and redshift-corrected UVES spectrum of SBS 0335-052W (ESO program 70.B-0717(A)).

that SBS 0335-052W consists of 4 regions (Fig. 2b). Our regions Nos. 1 and 2 correspond to the brightest western and eastern regions of Lipovetsky et al. (1999). The oxygen abundance of $12+\log \mathrm{O} / \mathrm{H}=7.22 \pm 0.07$ of the brightest region No. 1 (FORS) is consistent with the value $12+\log \mathrm{O} / \mathrm{H}=7.22 \pm$ 0.03 inferred by Lipovetsky et al. (1999). However, our oxygen abundance of $12+\log \mathrm{O} / \mathrm{H}=7.01 \pm 0.07$ for region No. 2 is significantly lower than that of $12+\log \mathrm{O} / \mathrm{H}=7.13 \pm 0.07$ obtained by Lipovetsky et al. (1999). The main reason for the difference is that Lipovetsky et al. (1999) did not observe the blue part of the spectrum in region No. 2 covering the emission line [O II] $\lambda 3727$ and assumed its flux relative to $\mathrm{H} \beta$ to be the same as that in region No. 1 . The relative flux [O II] $\lambda 3727 / \mathrm{H} \beta$ in this region is a factor of $\sim 2$ lower than that adopted by Lipovetsky et al. (1999) (see Table 2). Applying our value of [O II] $\lambda 3727 / \mathrm{H} \beta$ ratio to the Lipovetsky et al. (1999) observations, we obtain $12+\log \mathrm{O} / \mathrm{H}=7.04 \pm 0.07$, which is very close to the value for region No. 2 obtained from our data. The fainter regions Nos. 3 and 4 in SBS 0335-052W were not discussed in previous papers and for the first time, we derive element abundances in these regions. Thus, we confirm the very low oxygen abundance of region 1 . We also find that three of four $\mathrm{H}$ II regions have unprecedently low oxygen abundances of $12+\log \mathrm{O} / \mathrm{H}=7.01 \pm 0.07$ (region 2), $6.98 \pm 0.06$ (region 3 ), and $6.86 \pm 0.14$ (region 4 ), confirming our previous findings that this galaxy is the most metal-deficient emission-line galaxy known. We note, that [O III] $\lambda 4363$ emission line was not detected for region 3 . Therefore, the oxygen abundance in this region was derived using the semi-empirical method described by Izotov \& Thuan (2007). Similar to SBS 0335-052E, we find that the oxygen abundance in SBS 0335-052W varies from region to region. These variations are in the range from $12+\log \mathrm{O} / \mathrm{H}=$ 6.87 to 7.22 , which is even higher than the variations that we obtained for SBS 0335-052E.

If real, the oxygen abundance variations in both galaxies would argue in favour of self-enrichment by the fresh heavy elements synthesized during the present burst of star formation and slow mixing of these elements with the surrounding regions.
It is also possible that the [O III] $\lambda 4363 \AA$ emission line is enhanced by shocks and the effect is likely to be stronger in regions with weaker lines (Peimbert et al. 1991; Izotov et al. 1997a). Consequently, this enhancement would result in the underestimation of the oxygen abundance. However, this effect is difficult to take into account because of insufficient information about the shock contribution to ionization and heating of $\mathrm{H}$ II regions.

\subsection{Other heavy element abundances}

In Fig. 10, we show the abundance ratios $\log \mathrm{N} / \mathrm{O}$ (a), $\log \mathrm{Ne} / \mathrm{O}(\mathrm{b}), \log \mathrm{S} / \mathrm{O}$ (c), $\log \mathrm{Cl} / \mathrm{O}$ (d), $\log \mathrm{Ar} / \mathrm{O}$ (e), and $\log \mathrm{Fe} / \mathrm{O}$ (f) versus oxygen abundance $12+\log \mathrm{O} / \mathrm{H}$ for different $\mathrm{H}$ II regions in SBS 0335-052E and SBS 0335-052W, and compare them with data for a large sample of emission-line galaxies. Data for H II regions in SBS 0335-052E are shown by large filled circles and for H II regions in SBS 0335-052W by stars. The galaxies from comparison samples are shown by small symbols. We show by small filled circles data for galaxies collected to study the helium abundances in low-metallicity blue compact dwarf galaxies (the HeBCD sample, Izotov et al. 2004b; Izotov \& Thuan 2004), and by dots the galaxies from the SDSS DR3 sample (Izotov et al. 2006b). In each panel, the solar abundance ratio by Lodders (2003) is indicated by the large open circle and the associated error bar.

As for $\alpha$-elements, the ratios of $\mathrm{Ne}, \mathrm{S}$, Ar abundances to oxygen abundance, $\mathrm{Ne} / \mathrm{O}$ derived for different regions in SBS 0335-052E and SBS 0335-052W follow the trend of increasing $\mathrm{Ne} / \mathrm{O}$ with oxygen abundance found by Izotov et al. (2006b) for other low-metallicity emission-line galaxies (Fig. 10b). This trend is caused by oxygen depletion onto dust and implies that there is a small amount of depletion of oxygen in SBS 0335-052E and SBS 0335-052W despite the detection of dust in SBS 0335-052E (e.g., Thuan et al. 1999; Houck et al. 2004).

The S/O abundance ratio in both galaxies is close to the average value obtained for other low-metallicity emission-line 


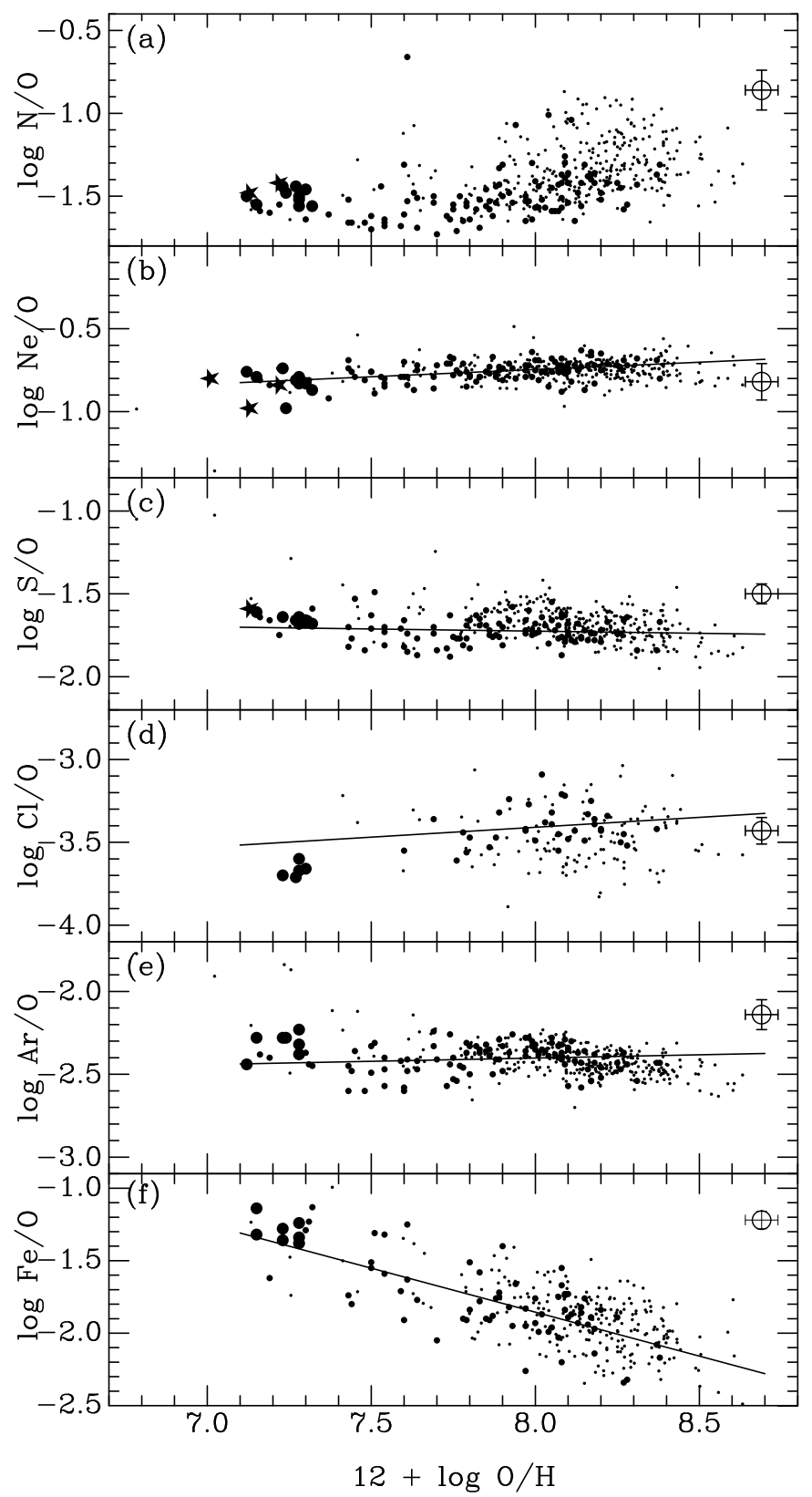

Fig. 10. $\log \mathrm{N} / \mathrm{O}$ a), $\log \mathrm{Ne} / \mathrm{O}$ b), $\log \mathrm{S} / \mathrm{O}$ c), $\log \mathrm{Cl} / \mathrm{O}$ d), $\log \mathrm{Ar} / \mathrm{O}$ e) and $\log \mathrm{Fe} / \mathrm{O}$ f) vs. oxygen abundance $12+\log \mathrm{O} / \mathrm{H}$ for the emissionline galaxies. Large filled circles show regions in SBS 0335-052E, large stars show regions in SBS 0335-052W. The galaxies from comparison samples are shown by small symbols. Small filled circles are galaxies from the HeBCD sample collected by Izotov et al. (2004b) and Izotov \& Thuan (2004) for the primordial He abundance determination, dots are the galaxies from the SDSS DR3 sample (Izotov et al. 2006b). The solar rations as compiled by Lodders (2003) are indicated by the large open circles and the associated error bars are shown.

galaxies (Fig. 10c). On the other hand, $\mathrm{Cl} / \mathrm{O}$ in SBS 0335$052 \mathrm{E}$ is systematically lower (Fig. 10d) and $\mathrm{Ar} / \mathrm{O}$ is systematically higher (Fig. 10e) than the averaged values obtained by Izotov et al. (2006b) for other low-metallicity galaxies. These differences imply that there is a metallicity dependence of the $\alpha$-element production by massive stars. The explosion energy of type II supernovae might play a role (Kobayashi et al. 2006). However, Izotov et al. (2006b) pointed out that abundance determinations for some elements such as $\mathrm{S}, \mathrm{Cl}$, and Ar could be uncertain because of the uncertainties in the rates of some atomic processes, e.g., rates of dielectronic recombination. Further analysis of larger samples of extremely low-metallicity galaxies is needed to clarify the reasons for $\mathrm{Cl} / \mathrm{O}$ and $\mathrm{Ar} / \mathrm{O}$ deviations in SBS 0335-052E from other galaxies.

The most prominent trend was found by Izotov et al. (2006b) for the Fe/O ratio. New data for SBS 0335-052E confirm and strengthen the previous result that $\mathrm{Fe}$ is depleted onto dust grains and that this effect depends on the galaxy metallicity. The depletion of Fe decreases with decreasing metallicity, and in the $\mathrm{H}$ II regions of SBS 0335-052E with the lowest metallicity, the depletion is the lowest.

Izotov \& Thuan (1999) and Izotov et al. (2006b) demonstrated that the dispersion in N/O in low metallicity BCDs with $12+\log \mathrm{O} / \mathrm{H}<7.5-7.6$ is very small with a plateau value of $\log \mathrm{N} / \mathrm{O} \sim-1.6$. However, in this paper, we find that $\mathrm{N} / \mathrm{O}$ in $\mathrm{H}$ II regions of SBS 0335-052E and SBS 0335-052W is higher than a plateau value implying that there is some increase in $\mathrm{N} / \mathrm{O}$ with decreasing oxygen abundance at $12+\log \mathrm{O} / \mathrm{H}<7.5$ (Fig. 10a). If true, this tendency would agree with studies of primary nitrogen production by low-metallicity rotating stars. For example, Meynet \& Maeder (2002) showed that the production of primary nitrogen is significantly higher in rapidly rotating stars of extremely low metallicity as compared to that in nonrotating stars. Although they considered stellar models with the heavy element mass fraction $Z=10^{-5}$, which is significantly lower than the values of $Z \sim 0.0002-0.0005$ in the $\mathrm{H}$ II regions of SBS 0335-052E and SBS 0335-052W, it is possible that the interstellar medium in these galaxies "memorizes" the chemical enrichment by the most metal-deficient stars.

\subsection{Helium abundance}

Helium $\left({ }^{4} \mathrm{He}\right)$ and some other light elements and isotopes such as $\mathrm{D},{ }^{3} \mathrm{He}$, and ${ }^{7} \mathrm{Li}$ were produced mainly within the first few minutes after the Big Bang and they can therefore be used to constrain cosmological models (e.g., Steigman 2005, 2006). Because of their extremely low metallicity, SBS 0335-052E and SBS 0335-052W are among the most suitable objects to use for the primordial He abundance determination. This is because the fraction of He produced by stars in these galaxies is less than $1 \%$ of the fraction of He synthesized during the primordial nucleosynthesis. Significant efforts have been made to determine the primordial He mass fraction $Y_{\mathrm{p}}$ from observations of lowmetallicity $\mathrm{H}$ II regions and the baryonic mass fraction of the Universe $\Omega_{\mathrm{b}}$. Because of the logarithmic dependence of $Y_{\mathrm{p}}$ on $\Omega_{\mathrm{b}}$, an accuracy of higher than $1 \%$ in $Y_{\mathrm{p}}$ is required to place constraints on the cosmological models. This high accuracy is feasible for the data obtained with the VLT for SBS 0335-052E and SBS 0335-052W (provided that the atomic data are known with sufficient accuracy, Porter et al. 2009). In this paper, we determine the He abundance following the method described by Izotov et al. (2007a).

There is now a general consensus that the accuracy of the primordial He abundance determination is limited, not so much by statistical uncertainties, but by our ability to account for systematic errors and biases (e.g., Olive \& Skillman 2004; Peimbert et al. 2007; Porter et al. 2009). There are many known effects to correct for when transforming observed He I line intensities into a He abundance. These effects are: (1) reddening; (2) underlying stellar absorption in the He I lines; (3) collisional excitation of the He I lines, which causes their intensities to deviate from their recombination values; (4) fluorescence of the He I lines, which also make their intensities deviate from their recombination values; (5) collisional excitation of the hydrogen lines (hydrogen 
is relevant because the helium abundance is calculated relative to that of hydrogen); (6) possible departures from case B in the emissivities of $\mathrm{H}$ and $\mathrm{He}$ I lines ${ }^{2}$; (7) the temperature structure of the H II region; and (8) its ionization structure. All of these corrections are at a level of a few percent except for effect (3), which may be much higher, exceeding $10 \%$, in the case of the He I $\lambda 5876$ emission line in hot and dense $\mathrm{H}$ II regions.

The derived $\mathrm{He}^{+}$abundance $y^{+}=\mathrm{He}^{+} / \mathrm{H}^{+}$depends on the adopted He I line emissivities. We considered two sets of He I emissivities: older values by Benjamin et al. (1999, 2002), which were used by Izotov \& Thuan (2004) [Benjamin et al. (2002) take into account both collisional and fluorescent enhancements], and more recent values by Porter et al. (2005), which were computed using improved radiative and collisional data. Following Izotov et al. (1994, 1997a) and Izotov \& Thuan (1998), we used the five strongest He I $\lambda 3889, \lambda 4471, \lambda 5876$, $\lambda 6678$, and $\lambda 7065$ emission lines to derive the weighted mean $\mathrm{He}^{+}$abundance $y_{\mathrm{wm}}^{+}$, where the weights were defined by the flux error of each He I emission line.

In addition to the emissivities, the derived $y^{+}$abundances also depend on a number of other parameters: the fraction $\Delta I(\mathrm{H} \alpha) / I(\mathrm{H} \alpha)$ of the $\mathrm{H} \alpha$ emission line flux due to collisional excitation; the electron number density $N_{\mathrm{e}}\left(\mathrm{He}^{+}\right)$; the electron temperature $T_{\mathrm{e}}\left(\mathrm{He}^{+}\right)$; the equivalent widths $E W_{\text {abs }}(\lambda 3889), E W_{\text {abs }}(\lambda 4471), E W_{\text {abs }}(\lambda 5876)$, $E W_{\text {abs }}(\lambda 6678)$, and $E W_{\text {abs }}(\lambda 7065)$ of He I stellar absorption lines; and the optical depth $\tau(\lambda 3889)$ of the He I $\lambda 3889$ emission line. To determine the most robust value of $y_{\mathrm{wm}}^{+}$, we use the Monte Carlo procedure described in Izotov \& Thuan (2004), randomly varying each of the above parameters within a specified range. First, we took into account collisional excitation effects for hydrogen. The fraction of the $\mathrm{H} \alpha$ flux produced by collisional excitation was randomly generated 100 times within an adopted range. The fraction of the $\mathrm{H} \beta$ emission line flux due to the collisional excitation was adopted to be three times less than that of the $\mathrm{H} \alpha$ flux. For each generated fraction, the fluxes of the $\mathrm{H} \alpha$ and $\mathrm{H} \beta$ lines caused by the collisional excitation were subtracted from the total observed fluxes and all emission line fluxes were then corrected for underlying stellar absorption (in the case of hydrogen lines) and interstellar extinction, and element abundances were calculated.

To calculate $y^{+}$, we varied $N_{\mathrm{e}}\left(\mathrm{He}^{+}\right)$simultaneously and randomly in the range $10-450 \mathrm{~cm}^{-3}, T_{\mathrm{e}}\left(\mathrm{He}^{+}\right)$in the range $(0.95-1.0) \times T_{\mathrm{e}}(\mathrm{O}$ III $)$, and $\tau(\lambda 3889)$ in the range $0-5$. We produced a total of $10^{5}$ of these realizations for every $\mathrm{H}$ II region, for a given fraction of $\mathrm{H} \alpha$ emission line flux created by collisional excitation. Thus, the total number of Monte Carlo realizations that we performed for each $\mathrm{H}$ II region was $100 \times 10^{5}=10^{7}$. As for the He I underlying stellar absorption, we followed prescriptions by Izotov et al. (2007a). We adopted fixed values of $E W_{\mathrm{abs}}(\lambda 4471)=$ $0.4 \AA, E W_{\mathrm{abs}}(\lambda 3889) / E W_{\mathrm{abs}}(\lambda 4471)=1.0, E W_{\mathrm{abs}}(\lambda 5876) /$ $E W_{\text {abs }}(\lambda 4471)=0.3, E W_{\text {abs }}(\lambda 6678) / E W_{\text {abs }}(\lambda 4471)=0.1$, and $E W_{\text {abs }}(\lambda 7065) / E W_{\text {abs }}(\lambda 4471)=0.1$.

For each H II region, we found the optimal solution for $y_{\mathrm{wm}}^{+}$ in the multi-parameter space defined above by minimizing the quantity

$\chi^{2}=\sum_{i}^{n} \frac{\left(y_{i}^{+}-y_{\mathrm{wm}}^{+}\right)^{2}}{\sigma^{2}\left(y_{i}^{+}\right)}$,

\footnotetext{
${ }^{2}$ Case B assumes that there exists a balance between the absorption and emission of photons in the resonant Lyman series transitions of hydrogen and helium and that there are no other processes.
}

where $y_{i}^{+}$is the $\mathrm{He}^{+}$abundance derived from the flux of the He I emission line labelled $i$, and $\sigma\left(y_{i}^{+}\right)$is the statistical error of $y_{i}^{+}$. The quantity $y_{\mathrm{wm}}^{+}$is the weighted mean of the $\mathrm{He}^{+}$abundance given by the equation

$y_{\mathrm{wm}}^{+}=\frac{\sum_{i}^{k} y_{i}^{+} / \sigma^{2}\left(y_{i}^{+}\right)}{\sum_{i}^{k} 1 / \sigma^{2}\left(y_{i}^{+}\right)}$.

We used all five He I emission lines to calculate $\chi^{2}$ (i.e., $n=5$ ), but only three lines, He I $\lambda 4471, \lambda 5876$, and $\lambda 6678$ to compute $y_{\mathrm{wm}}^{+}(k=3)$. This is because the fluxes of the He I $\lambda 3889$ and $\lambda 7065$ emission lines are more uncertain than those of the other three He I emission lines.

Additionally, in those cases when the nebular He II $\lambda 4686$ emission line was detected, we added the abundance of doubly ionized helium $y^{2+} \equiv \mathrm{He}^{2+} / \mathrm{H}^{+}$to $y^{+}$. Although the $\mathrm{He}^{2+}$ zone is hotter than the $\mathrm{He}^{+}$zone, we adopted $T_{\mathrm{e}}\left(\mathrm{He}^{2+}\right)=T_{\mathrm{e}}\left(\mathrm{He}^{+}\right)$. This assumption has only a minor effect on the $y$ value, because $y^{2+}$ is small $\left(\leq 3 \%\right.$ of $\left.y^{+}\right)$in all cases. Then the total He abundance was derived to be

$y=I C F\left(\mathrm{He}^{+}+\mathrm{He}^{2+}\right) \times\left(y^{+}+y^{2+}\right)$,

where $I C F\left(\mathrm{He}^{+}+\mathrm{He}^{2+}\right)$ is the ionization correction factor, adopted from Izotov et al. (2007a). The He mass fraction was obtained from

$Y=\frac{4 y(1-Z)}{1+4 y}$

where $Z$ is the heavy element mass fraction. We adopted $Z=$ $a(\mathrm{O} / \mathrm{H})$ in Eq. (4), where $a=18.2$ was obtained by assuming an oxygen mass fraction of $O=0.66 Z$ for a heavy element mass fraction $Z=0.001$ (Maeder 1992). Some authors use slightly different values of $a$. In particular, Pagel et al. (1992) adopted $a=20$. These differences introduced a tiny effect on the $Y$ value in $\mathrm{H}$ II regions with $\mathrm{O} / \mathrm{H} \lesssim 10^{-4}$, resulting in a difference of $\lessgtr 0.02 \%$ in $Y$, if our value of $a$ is adopted instead of that by Pagel et al. (1992).

The results of our determinations of the He abundance in different regions of SBS 0335-052E and SBS 0335-052W corresponding to minimum of $\chi^{2}$ for two different sets of emissivities are shown in Table 8 . It can be seen that the derived He mass fractions $Y$ are very consistent (within $\lesssim 1 \%$ ) in all $\mathrm{H}$ II regions, while the statistical errors $1-5 \%$ of the $Y$ values are comparable to those obtained by other authors for different galaxies (see e.g., Peimbert et al. 2007). The weighted mean $Y$ derived from all observations shown in Table 8 is equal to $Y_{\mathrm{wm}}=0.2485 \pm 0.0012$ for the emissivities derived by Benjamin et al. $(1999,2002)$ and $Y_{\mathrm{wm}}=0.2514 \pm 0.0012$ for Porter et al. (2005) emissivities. These values are very close to the primordial He abundance since the considered H II regions are of very low-metallicity. They are obtained by taking into account almost all systematic effects. The largest systematic effect is collisional excitation of He I emission lines. In the case of SBS 0335-052E Nos. $1+2$ this effect could increase the intensity of the strongest He I $\lambda 5876$ emission line by up to $10 \%$ relative to the recombination value. Collisional excitation of the He I $\lambda 7065$ emission line has an even stronger effect, of $\sim 50 \%$. If not taken into account, this systematic effect can produce the weighted mean He mass fraction of 0.266 , or a value $\sim 6 \%$ higher than the value of 0.251 in Table 8 for Porter et al. (2005) emissivities. This difference is much higher than the errors of $\sim 1-3 \%$ for $Y_{\mathrm{p}}$ obtained by different authors. However, since these systematic effects are taken into account in our $Y$ determination, they are not included in the 

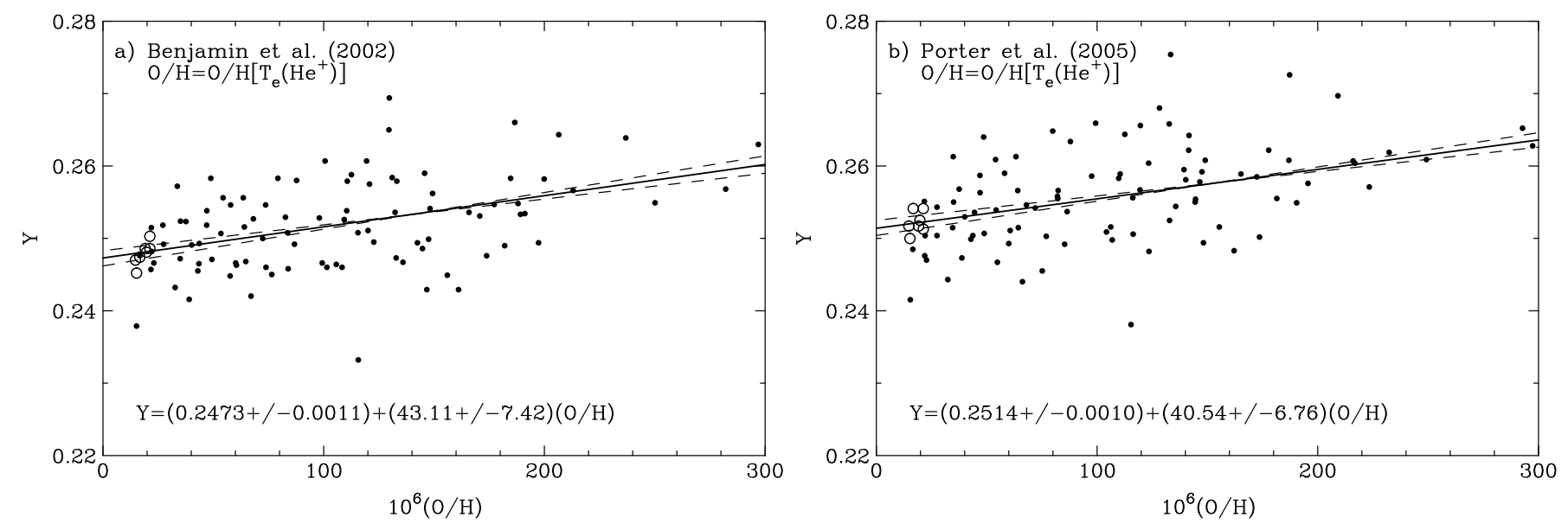

Fig. 11. Linear regressions of the helium mass fraction $Y$ vs. oxygen abundance for the sample consisting of H II regions in SBS 0335-052E and SBS 0335-052W (open circles, this paper), and HeBCD H II regions (dots) from Izotov et al. (2007a). Solid lines are linear regressions obtained by a likelihood method, and dashed lines are their $1 \sigma$ alternatives. The $Y \mathrm{~s}$ in $\mathbf{a}$ ) and $\mathbf{b}$ ) are derived with the He I emissivities from Benjamin et al. $(1999,2002)$ and Porter et al. (2005), respectively. The oxygen abundance is derived adopting $T_{\mathrm{e}}\left(\mathrm{He}^{+}\right)$.

error budget. An exception is the differences between two different sets of He I emissivities by Benjamin et al. $(1999,2002)$ and Porter et al. (2005). Treating the different He I line emissivities as systematics affecting the $Y_{\mathrm{wm}}$ determination, we obtain $Y_{\mathrm{wm}}=$ $0.2500 \pm 0.0012$ (stat.) \pm 0.0015 (syst.).

We also rederive the primordial He abundance from linear regressions to $Y-\mathrm{O} / \mathrm{H}$ for the large sample of low-metallicity $\mathrm{H}$ II regions from Izotov et al. (2007a), including also our values for SBS 0335-052E and SBS 0335-052W from the present study. These regressions are obtained by taking into account the statistical errors in $Y$ and $\mathrm{O} / \mathrm{H}$ for every object and are shown in Fig. 11 for two sets of emissivities, Benjamin et al. (1999, 2002) (Fig. 11a) and Porter et al. (2005) (Fig. 11b). In this figure, we indicate by dots 93 observations of $77 \mathrm{H}$ II regions from the HeBCD sample (Izotov \& Thuan 2004; Izotov et al. 2007a) and by open circles different $\mathrm{H}$ II regions in SBS 0335-052E and SBS 0335-052W. Solid lines are linear regressions obtained by a likelihood method, and the dashed lines are $1 \sigma$ alternatives to these regressions. We obtain $Y_{\mathrm{p}}=0.2473 \pm 0.0011$ for the Benjamin et al. $(1999,2002)$ emissivities and $Y_{\mathrm{p}}=0.2514 \pm$ 0.0010 for the Porter et al. (2005) emissivities. Despite the statistical errors of $Y$ for the $\mathrm{H}$ II regions in our sample being comparable to those derived in other studies, the statistical errors of $Y_{\mathrm{p}}$ are significantly lower than those derived by e.g., Olive \& Skillman (2004), Fukugita \& Kawasaki (2006), and Peimbert et al. (2007). This difference is present because we use the largest sample of $\sim 100 \mathrm{H}$ II regions to determine $Y_{\mathrm{p}}$ compared to 30 or even only $6 \mathrm{H}$ II regions in other studies. The error in $Y_{\mathrm{p}}$ scales as $\sim 1 / \sqrt{N}$, where $N$ is the number of the objects used for the primordial He abundance determination, resulting in the reduction in the $Y_{\mathrm{p}}$ error by a factor of 2-4 for our large sample. Our values of $Y_{\mathrm{p}}$ are only slightly below ( $\left.\lesssim 1 \%\right)$ the values of $Y_{\mathrm{wm}}$ obtained for the BCD system SBS 0335-052 with the respective emissivities, but they are consistent within the errors. The value of $Y_{\mathrm{p}}$ obtained with the Benjamin et al. (1999, 2002) emissivities is in good agreement with the value of $Y_{\mathrm{p}}=$ $0.2482_{-0.0004}^{+0.0003} \pm 0.0006$ (syst) inferred by Spergel et al. (2007) from the WMAP data analysis. On the other hand, the $Y_{\mathrm{p}}$ derived with the Porter et al. (2005) emissivities is slightly above the WMAP value. If real, this higher value would suggest some deviations from the Standard Big Bang Nucleosynthesis model.
On the other hand, treating the different He I line emissivities as systematics affecting the $Y_{\mathrm{p}}$ determination, we obtain $Y_{\mathrm{p}}=$ $0.2493 \pm 0.0011$ (stat.) \pm 0.0020 (syst.), which agrees with the $Y_{\mathrm{p}}$ derived from the WMAP data analysis.

\section{Summary}

We have presented our analysis of archival VLT/FORS and VLT/UVES spectroscopic observations of a system of two extremely low-metallicity blue compact dwarf (BCD) galaxies, SBS 0335-052E and SBS 0335-052W. Some of these spectroscopic data are of the highest signal-to-noise ratio, highest spectral resolution and highest spatial resolution obtained up to date for this system of galaxies since its first spectroscopic observations, 20 years ago (Izotov et al. 1990a,b,c).

Our main results are as follows:

1. The oxygen abundance in different regions of both galaxies is extremely low. We find that the oxygen abundance $12+\log \mathrm{O} / \mathrm{H}$ in SBS 0335-052E varies in the range 7.12-7.32, while in SBS 0335-052W, it varies in the range 6.86-7.22. Thus, SBS 0335-052W holds the record as the most metal-deficient emission-line galaxy known, supporting and strengthening previous findings by Izotov et al. (2005) and Papaderos et al. (2006). We suggest that the oxygen abundance variations in both galaxies are likely to be real and imply incomplete mixing of the interstellar medium and the chemical element self-enrichment of $\mathrm{H}$ II regions by the present bursts of star formation.

2. We derive the He mass fraction $Y$ in different $\mathrm{H}$ II regions of SBS 0335-052E and SBS 0335-052W using Monte Carlo simulations and taking into account systematic effects. We find that the He mass fraction in all studied $\mathrm{H}$ II regions varies only a little and, depending on the adopted He I line emissivities, has a weighted mean value $Y_{\mathrm{wm}}$ of $0.2485 \pm$ 0.0012 with the Benjamin et al. $(1999,2002)$ emissivities and of $0.2514 \pm 0.0012$ with the Porter et al. (2005) emissivities. Using the data of the present paper for SBS 0335-052E and SBS 0335-052W in connection with the HeBCD sample of low-metallicity BCDs by Izotov \& Thuan (2004) and Izotov et al. (2007a), we rederive the primordial He mass 
fraction $Y_{\mathrm{p}}$ from linear regressions to $Y$ versus $\mathrm{O} / \mathrm{H}$. We determine a value of $Y_{\mathrm{p}}=0.2473 \pm 0.0011$ using the Benjamin et al. $(1999,2002) \mathrm{He}$ I emissivities and of $0.2514 \pm$ 0.0010 using the Porter et al. (2005) He I emissivities. The former value of $Y_{\mathrm{p}}$ is consistent with the value inferred from the WMAP data analysis, implying the validity of the Standard Big Bang Nucleosynthesis (SBBN) model, while the latter value of $Y_{\mathrm{p}}$ is higher by more than $2 \sigma$, implying that some deviations from the SBBN could be present. Treating the different He I line emissivities as systematics affecting the $Y_{\mathrm{wm}}$ and $Y_{\mathrm{p}}$ determinations, we obtain $Y_{\mathrm{wm}}=0.2500 \pm 0.0012$ (stat.) \pm 0.0015 (syst.), and $Y_{\mathrm{p}}=0.2493 \pm 0.0011$ (stat.) \pm 0.0020 (syst.). Both $Y_{\mathrm{wm}}$ and $Y_{\mathrm{p}}$ values within the errors agree with the $Y_{\mathrm{p}}$ value from the WMAP data analysis.

3 . The abundance ratios of the $\alpha$-elements to oxygen in both galaxies follow the trends found in previous studies of lowmetallicity emission-line galaxies. In particular, new data confirm findings by Izotov et al. (2006b) that Ne/O increases with increasing oxygen abundance, implying a higher depletion of oxygen in higher-metallicity galaxies. We find that $\mathrm{S} / \mathrm{O}$ in both galaxies is close to the average value obtained for other low-metallicity emission-line galaxies. On the other hand, $\mathrm{Cl} / \mathrm{O}$ in SBS 0335-052E is lower than the average value for other low-metallicity emission-line galaxies, while $\mathrm{Ar} / \mathrm{O}$ is higher than the average value. These differences could be due to the metallicity-dependent yields of $\alpha$-elements synthesized by massive stars. However, it is also possible that differences are caused by uncertainties in the atomic data for some elements (e.g., uncertainties in the dielectronic recombination rates), which infer incorrect abundances of sulfur, chlorine, and argon.

4. The measurement of Fe/O in SBS 0335-052E is among the highest values found in emission-line galaxies and follows the general trend of decreasing $\mathrm{Fe} / \mathrm{O}$ with increasing oxygen abundance implying iron depletion onto dust (Izotov et al. 2006b). Despite the detection of dust in SBS 0335-052E by Thuan et al. (1999) and Houck et al. (2004), the high gasphase $\mathrm{Fe} / \mathrm{O}$ abundance ratio suggests that iron is hardly depleted in the ionized medium of this galaxy.

5. We find that N/O in SBS 0335-052E and SBS 0335-052W is slightly above the plateau value found by Izotov \& Thuan (1999) and Izotov et al. (2006b) for extremely lowmetallicity emission-line galaxies. There is a tendency for $\mathrm{N} / \mathrm{O}$ increasing with decreasing oxygen abundance in extremely low-metallicity galaxies with $12+\log \mathrm{O} / \mathrm{H}<7.6$, probably because of an enhanced production of primary nitrogen by lower-metallicity rapidly rotating stars (Meynet \& Maeder 2002).

Acknowledgements. N.G.G. and Y.I.I. thank the hospitality of the Max Planck Institute for Radioastronomy (Bonn), and acknowledge support through DFG grant No. FR 325/57-1. P.P. thanks the Department of Astronomy and Space Physics at Uppsala University for its warm hospitality. This research was partially funded by project grant AYA2007-67965-C03-02 of the Spanish Ministerio de Ciencia e Innovacion.

\section{References}

Benjamin, R. A., Skillman, E. D., \& Smits, D. P. 1999, ApJ, 514, 307 Benjamin, R. A., Skillman, E. D., \& Smits, D. P. 2002, ApJ, 569, 288
Fricke, K. J., Izotov, Y. I., Papaderos, P., Guseva, N. G., \& Thuan, T. X. 2001, AJ, 121, 169

Fukugita, M., \& Kawasaki, M. 2006, ApJ, 646, 691

Guseva, N. G., Izotov, Y. I., \& Thuan, T. X. 2000, ApJ, 531, 776

Houck, J. R., Charmandaris, V., Brandl, B. R., et al. 2004, ApJS, 154, 211

Hunt, L. K., Vanzi, L., \& Thuan, T. X. 2001, A\&A, 377, 66

Izotov, Y. I., \& Thuan, T. X. 1998, ApJ, 500, 188

Izotov, Y. I., \& Thuan, T. X. 1999, ApJ, 511, 639

Izotov, Y. I., \& Thuan, T. X. 2004, ApJ, 602, 200

Izotov, Y. I., \& Thuan, T. X. 2007, ApJ, 665, 1115

Izotov, Y. I., Guseva, N. G., Lipovetsky, V. A., Kniazev, A. Y., \& Stepanian, J. A. 1990a, Nature, 343, 238

Izotov, Y. I., Lipovetsky, V. A., Guseva, N. G., \& Kniazev, A. Y. 1990b, SvA, 34, 343

Izotov, Y. I., Lipovetsky, V. A., Guseva, N. G., \& Stepanian, J. A. 1990c, SvA Lett., 16, 258

Izotov, Y. I., Thuan, T. X., \& Lipovetsky, V. A. 1994, ApJ, 435, 647 Izotov, Y. I., Thuan, T. X., \& Lipovetsky, V. A. 1997a, ApJS, 108, 1 Izotov, Y. I., Lipovetsky, V. A., Chaffee, F. H., et al. 1997b, ApJ, 476, 698 Izotov, Y. I., Chaffee, F. H., Foltz, C. B., et al. 1999, ApJ, 527, 757 Izotov, Y. I., Chaffee, F. H., \& Schaerer, D. 2001, A\&A, 378, L45 Izotov, Y. I., Noeske, K. G., Guseva, N. G., et al. 2004a, A\&A, 415, L27

Izotov, Y. I., Stasińska, G., Guseva, N. G., \& Thuan, T. X. 2004b, A\&A, 415, 87 Izotov, Y. I., Thuan, T. X., \& Guseva, N. G. 2005, ApJ, 415, 87

Izotov, Y. I., Schaerer, D., Blecha, A., et al. 2006a, A\&A, 459, 71

Izotov, Y. I., Stasińska, G., Meynet, G., Guseva, N. G., \& Thuan, T. X. 2006b, A\&A, 448, 955

Izotov, Y. I., Thuan, T. X., \& Stasińska, G. 2007a, ApJ, 662, 15

Izotov, Y. I., Thuan, T. X., \& Guseva, N. G. 2007b, ApJ, 671, 1297

Kobayashi, C., Umeda, H., Nomoto, K., Tominaga, N., \& Ohkubo, T. 2006, ApJ, 653, 1145

Kunth, D., \& Sargent, W. L. W. 1986, ApJ, 300, 496

Legrand, F., Kunth, D., Roy, J.-R., Mas-Hesse, J. M., \& Walsh, J. R. 1997, A\&A, 326, L17

Lipovetsky, V. A., Chaffee, F. H., Izotov, Y. I., et al. 1999, ApJ, 519, 177

Lodders, K. 2003, ApJ, 591, 1220

Maeder, A. 1992, A\&A, 264, 105

Melnick, J., Heydari-Malayeri, M., \& Leisy, P. 1992, A\&A, 253, 16

Meynet, G., \& Maeder, A. 2002, A\&A, 390, 561

Olive, K. A., \& Skillman, E. 2004, ApJ, 617, 29

Pagel, B. E. J., Simonson, E. A., Terlevich, R. J., \& Edmunds, M. G. 1992, MNRAS, 255, 325

Papaderos, P., Izotov, Y. I., Fricke, K. J., Thuan, T. X., \& Guseva, N. G. 1998, A\&A, 338, 43

Papaderos, P., Izotov, Y. I., Guseva, N. G., Thuan, T. X., \& Fricke, K. J. 2006, A\&A, 454, 119

Peimbert, M., Sarmiento, A., \& Fierro, J. 1991, PASP, 103, 815

Peimbert, M., Luridiana, V., \& Peimbert, A. 2007, ApJ, 666, 636

Péquignot, D., Petitjean, P., \& Boisson, C. 1991, A\&A, 251, 680

Piljugin, L. S. 1992, A\&A, 260, 58

Porter, R. L., Bauman, R. P., Ferland, G. J., \& MacAdam, K. B. 2005, ApJ, 622, L73

Porter, R. L., Ferland, G. J., MacAdam, K. B., \& Storey, P. J. 2009, MNRAS, 393, L36

Pustilnik, S. A., Lipovetsky, V. A., Izotov, Y. I., et al. 1997, Ast. L., 23, 308

Pustilnik, S. A., Brinks, E., Thuan, T. X., Lipovetsky, V. A., \& Izotov, Y. I. 2001, AJ, 121, 1413

Pustilnik, S. A., Kniazev, A. Yu., \& Pramskij, A. G. 2005, A\&A, 443, 91

Schaerer, D., \& Vacca, W. D. 1998, ApJ, 497, 618

Spergel, D. N., Bean, R., Doré, O., et al. 2007, ApJS, 170, 377

Steigman, G. 2005, Phys. Scr. T, 121, 142

Steigman, G. 2006, Int. J. Mod. Phys. E, 15, 1

Thompson, R. I., Sauvage, M., Kennicutt, R. C., \& Engelbracht, C. W. 2006, ApJ, 638, 176

Thompson, R. I., Sauvage, M., Kennicutt, R. C., et al. 2008 [arXiv:0810.2540v1]

Thuan, T. X., \& Izotov, Y. I. 1997, ApJ, 489, 623

Thuan, T. X., \& Izotov, Y. I. 2005, ApJS, 161, 240

Thuan, T. X., Izotov, Y. I., \& Lipovetsky, V. A. 1997, ApJ, 477, 661

Thuan, T. X., Sauvage, M., \& Madden, S. 1999, ApJ, 516, 783

Thuan, T. X., Bauer, F. E., Papaderos, P., \& Izotov, Y. I. 2004, ApJ, 606, 213

Vanzi, L., Hunt, L. K., Thuan, T. X., \& Izotov, Y. I. 2000, A\&A, 363, 493

Whitford, A. E. 1958, AJ, 63, 201 
Y. I. Izotov et al.: SBS 0335-052E+W: deep VLT/FORS+UVES spectroscopy, Online Material p 1

Table 1. Extinction-corrected emission line fluxes (low-resolution FORS observations).

\begin{tabular}{|c|c|c|}
\hline \multirow[b]{2}{*}{ Line } & \multicolumn{2}{|c|}{$0335-052 \mathrm{E}$} \\
\hline & Nos. $1+2$ & No. 7 \\
\hline 3727 [O II] & $23.49 \pm 0.55$ & $24.82 \pm 2.84$ \\
\hline $3750 \mathrm{H} 12$ & $3.90 \pm 0.42$ & $\ldots$ \\
\hline 3771 H11 & $4.60 \pm 0.43$ & $\ldots$ \\
\hline 3797 H10 & $5.89 \pm 0.37$ & $\ldots$ \\
\hline $3820 \mathrm{He}$ I & $1.04 \pm 0.21$ & \\
\hline 3835 H9 & $8.10 \pm 0.35$ & $9.34 \pm 2.15$ \\
\hline 3869 [Ne III] & $24.46 \pm 0.43$ & $17.48 \pm 1.39$ \\
\hline $3889 \mathrm{He} \mathrm{I} \mathrm{+} \mathrm{H8}$ & $16.77 \pm 0.40$ & $22.70 \pm 1.85$ \\
\hline $3968[\mathrm{Ne}$ III] + H7 & $24.22 \pm 0.47$ & $22.06 \pm 1.65$ \\
\hline $4026 \mathrm{He} \mathrm{I}$ & $1.64 \pm 0.11$ & $\ldots$ \\
\hline 4069 [S II] & $0.41 \pm 0.08$ & ... \\
\hline 4076 [S II] & & \\
\hline $4101 \mathrm{H} \delta$ & $26.30 \pm 0.47$ & $27.73 \pm 1.63$ \\
\hline 4227 [Fe IV] & $1.21 \pm 0.45$ & \\
\hline $4340 \mathrm{H} \gamma$ & $47.38 \pm 0.74$ & $47.06 \pm 1.60$ \\
\hline 4363 [O III] & $11.22 \pm 0.19$ & $6.88 \pm 0.58$ \\
\hline $4388 \mathrm{He} \mathrm{I}$ & $0.40 \pm 0.08$ & \\
\hline $4471 \mathrm{He} \mathrm{I}$ & $3.90 \pm 0.10$ & $3.76 \pm 0.52$ \\
\hline 4658 [Fe III] & $0.37 \pm 0.05$ & $\ldots$ \\
\hline 4686 He II & $1.16 \pm 0.06$ & $\ldots$ \\
\hline 4711 [Ar IV] + He I & $1.68 \pm 0.07$ & $\ldots$ \\
\hline 4740 [Ar IV] & $0.86 \pm 0.06$ & \\
\hline $4861 \mathrm{H} \beta$ & $100.00 \pm 1.46$ & $100.00 \pm 2.00$ \\
\hline $4921 \mathrm{He}$ I & $0.96 \pm 0.05$ & \\
\hline 4959 [O III] & $109.47 \pm 1.58$ & $68.06 \pm 1.25$ \\
\hline 4988 [Fe III] & $0.47 \pm 0.05$ & \\
\hline 5007 [O III] & $315.18 \pm 4.54$ & $198.83 \pm 3.33$ \\
\hline $5199[\mathrm{~N} \mathrm{I}]$ & $0.26 \pm 0.05$ & $\ldots$ \\
\hline $5518[\mathrm{Cl} \mathrm{III}]$ & $0.14 \pm 0.03$ & $\ldots$ \\
\hline 5538 [Cl III] & $0.08 \pm 0.03$ & \\
\hline $5876 \mathrm{He} \mathrm{I}$ & $10.82 \pm 0.17$ & $9.22 \pm 0.39$ \\
\hline 6300 [O I] & $0.75 \pm 0.04$ & $\ldots$ \\
\hline 6312 [S III] & $0.61 \pm 0.04$ & $\ldots$ \\
\hline $6364[\mathrm{O} \mathrm{I}]$ & $0.24 \pm 0.03$ & \\
\hline $6563 \mathrm{H} \alpha$ & $273.80 \pm 4.28$ & $273.83 \pm 4.90$ \\
\hline 6583 [N II] & $0.90 \pm 0.04$ & $1.01 \pm 0.31$ \\
\hline 6678 He I & $2.57 \pm 0.06$ & $2.74 \pm 0.29$ \\
\hline 6716 [S II] & $1.74 \pm 0.05$ & $2.59 \pm 0.32$ \\
\hline $6731[\mathrm{~S} \mathrm{II}]$ & $1.65 \pm 0.05$ & $2.48 \pm 0.35$ \\
\hline $7065 \mathrm{He} \mathrm{I}$ & $4.29 \pm 0.09$ & $1.89 \pm 0.29$ \\
\hline 7136 [Ar III] & $1.52 \pm 0.05$ & $1.07 \pm 0.24$ \\
\hline $7281 \mathrm{He} \mathrm{I}$ & $0.62 \pm 0.04$ & 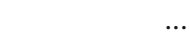 \\
\hline 7320 [O II] & $0.58 \pm 0.04$ & 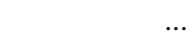 \\
\hline 7330 [O II] & $0.43 \pm 0.04$ & \\
\hline$C(\mathrm{H} \beta)$ & 0.180 & 0.140 \\
\hline$E W(\mathrm{H} \beta))^{a}$ & 351 & 298 \\
\hline$F(\mathrm{H} \beta)^{b}$ & 222.2 & 16.0 \\
\hline$E W(\mathrm{abs})$ & 2.5 & 4.2 \\
\hline
\end{tabular}

${ }^{a}$ in $\AA{ }^{b}$ in units $10^{-16} \mathrm{erg} \mathrm{s}^{-1} \mathrm{~cm}^{-2}$. 
Y. I. Izotov et al.: SBS 0335-052E+W: deep VLT/FORS+UVES spectroscopy, Online Material p 2

Table 2. Extinction-corrected emission line fluxes (high-resolution FORS observations).

\begin{tabular}{|c|c|c|c|c|c|c|}
\hline \multirow[b]{2}{*}{ Line } & \multicolumn{2}{|c|}{$0335-052 \mathrm{E}$} & \multicolumn{4}{|c|}{$0335-052 \mathrm{~W}$} \\
\hline & Nos. $1+2$ & No. 7 & No. 1 & No. 2 & No. 3 & No. 4 \\
\hline 3727 [O II] & $21.81 \pm 0.34$ & $24.98 \pm 0.53$ & $75.20 \pm 2.55$ & $30.15 \pm 2.20$ & $79.16 \pm 7.95$ & $41.95 \pm 4.46$ \\
\hline 3750 H12 & $3.08 \pm 0.07$ & $5.84 \pm 0.49$ & $\ldots$ & $\ldots$ & $\ldots$ & $\ldots$ \\
\hline $3771 \mathrm{H} 11$ & $3.92 \pm 0.08$ & $6.32 \pm 0.45$ & $\ldots$ & $\ldots$ & $\ldots$ & $\ldots$ \\
\hline $3797 \mathrm{H} 10$ & $5.39 \pm 0.10$ & $7.28 \pm 0.43$ & $5.65 \pm 0.11$ & $9.14 \pm 1.96$ & $\ldots$ & $\ldots$ \\
\hline $3820 \mathrm{He} \mathrm{I}$ & $1.06 \pm 0.04$ & $0.91 \pm 0.19$ & & $\ldots$ & $\ldots$ & $\ldots$ \\
\hline 3835 H9 & $7.30 \pm 0.13$ & $9.16 \pm 0.39$ & $7.02 \pm 0.13$ & $9.96 \pm 1.81$ & $\ldots$ & $\ldots$ \\
\hline 3869 [Ne III] & $26.98 \pm 0.41$ & $15.86 \pm 0.35$ & $11.31 \pm 0.98$ & $12.43 \pm 1.07$ & $\ldots$ & $\ldots$ \\
\hline $3889 \mathrm{He} \mathrm{I}+\mathrm{H} 8$ & $16.96 \pm 0.26$ & $20.75 \pm 0.48$ & $19.92 \pm 1.02$ & $24.69 \pm 1.61$ & $\ldots$ & $24.83 \pm 3.90$ \\
\hline 3968 [Ne III] + H7 & $25.56 \pm 0.39$ & $23.30 \pm 0.50$ & $13.52 \pm 0.96$ & $23.38 \pm 1.51$ & $\ldots$ & $25.28 \pm 4.25$ \\
\hline $4026 \mathrm{He} \mathrm{I}$ & $1.79 \pm 0.04$ & $1.94 \pm 0.19$ & $\ldots$ & $\ldots$ & $\ldots$ & $\ldots$ \\
\hline 4069 [S II] & $0.39 \pm 0.03$ & $\ldots$ & $\ldots$ & $\ldots$ & $\ldots$ & $\ldots$ \\
\hline 4076 [S II] & & & & $\ldots$ & $\ldots$ & $\ldots$ \\
\hline $4101 \mathrm{H} \delta$ & $27.11 \pm 0.40$ & $27.10 \pm 0.52$ & $26.61 \pm 1.09$ & $27.17 \pm 1.51$ & $32.76 \pm 6.79$ & $24.80 \pm 3.80$ \\
\hline 4227 [Fe IV] & $0.25 \pm 0.04$ & & $\ldots$ & & $\ldots$ & \\
\hline $4340 \mathrm{H} \gamma$ & $47.61 \pm 0.69$ & $46.39 \pm 0.76$ & $45.94 \pm 1.28$ & $46.85 \pm 1.50$ & $44.10 \pm 4.96$ & $43.27 \pm 2.76$ \\
\hline 4363 [O III] & $11.33 \pm 0.17$ & $6.15 \pm 0.18$ & $3.33 \pm 0.58$ & $5.09 \pm 0.72$ & $\ldots$ & $2.51 \pm 0.80$ \\
\hline 4388 He I & $0.40 \pm 0.02$ & & $\ldots$ & $\ldots$ & $\ldots$ & $\ldots$ \\
\hline $4471 \mathrm{He} \mathrm{I}$ & $3.77 \pm 0.06$ & $3.54 \pm 0.13$ & $3.55 \pm 0.32$ & $4.52 \pm 0.71$ & $\ldots$ & $\ldots$ \\
\hline 4658 [Fe III] & $0.32 \pm 0.02$ & $0.40 \pm 0.09$ & $\ldots$ & $\ldots$ & $\ldots$ & $\ldots$ \\
\hline 4686 He II & $1.33 \pm 0.03$ & $1.33 \pm 0.14$ & $\ldots$ & $\ldots$ & $\ldots$ & .. \\
\hline 4711 [Ar IV] + He I & $1.72 \pm 0.03$ & $0.80 \pm 0.09$ & $\ldots$ & $\ldots$ & $\ldots$ & ... \\
\hline $4740[\mathrm{Ar}$ IV $]$ & $0.92 \pm 0.02$ & $0.44 \pm 0.12$ & $\ldots$ & $\ldots$ & $\ldots$ & $\ldots$ \\
\hline $4861 \mathrm{H} \beta$ & $100.00 \pm 1.42$ & $100.00 \pm 1.51$ & $100.00 \pm 2.01$ & $100.00 \pm 2.34$ & $100.00 \pm 5.42$ & $100.00 \pm 3.40$ \\
\hline $4921 \mathrm{He} \mathrm{I}$ & $0.91 \pm 0.02$ & $0.87 \pm 0.10$ & & & & \\
\hline 4959 [O III] & $101.50 \pm 1.44$ & $64.87 \pm 0.99$ & $43.23 \pm 1.02$ & $49.24 \pm 1.33$ & $22.91 \pm 2.56$ & $23.50 \pm 1.43$ \\
\hline 4988 [Fe III] & $0.38 \pm 0.01$ & $0.60 \pm 0.08$ & $\ldots$ & $\quad \ldots$ & $\ldots$ & $\ldots$ \\
\hline 5007 [O III] & $303.19 \pm 4.31$ & $194.48 \pm 2.89$ & $130.20 \pm 2.52$ & $147.95 \pm 3.21$ & $71.23 \pm 3.74$ & $75.37 \pm 2.42$ \\
\hline $5016 \mathrm{He}$ I & $2.09 \pm 0.03$ & $2.06 \pm 0.10$ & $2.99 \pm 0.41$ & $2.50 \pm 0.58$ & $\ldots$ & $\ldots$ \\
\hline 5199 [N I] & $0.28 \pm 0.01$ & $\ldots$ & $\ldots$ & $\ldots$ & $\ldots$ & $\ldots$ \\
\hline 5518 [Cl III] & $0.09 \pm 0.02$ & $\ldots$ & $\ldots$ & $\ldots$ & $\ldots$ & $\ldots$ \\
\hline 5538 [Cl III] & $0.07 \pm 0.02$ & $\ldots$ & $\ldots$ & $\ldots$ & $\ldots$ & ... \\
\hline 5755 [N II] & $0.06 \pm 0.01$ & $\ldots$ & $\ldots$ & $\ldots$ & $\ldots$ & $\ldots$ \\
\hline $5876 \mathrm{He} \mathrm{I}$ & $10.48 \pm 0.16$ & $9.09 \pm 0.22$ & $9.02 \pm 0.75$ & $10.34 \pm 0.87$ & $\ldots$ & $\ldots$ \\
\hline 6300 [O I] & $0.69 \pm 0.02$ & $0.75 \pm 0.12$ & $\ldots$ & $\ldots$ & $\ldots$ & $\ldots$ \\
\hline 6312 [S III] & $0.59 \pm 0.02$ & $0.51 \pm 0.12$ & $\ldots$ & $\ldots$ & $\ldots$ & ... \\
\hline 6364 [O I] & $0.22 \pm 0.01$ & $\cdots$ & $\ldots$ & $\ldots$ & $\ldots$ & ... \\
\hline 6548 [N II] & $0.28 \pm 0.02$ & & & & . & $\ldots$ \\
\hline $6563 \mathrm{H} \alpha$ & $272.75 \pm 4.20$ & $274.56 \pm 4.43$ & $279.19 \pm 5.65$ & $274.11 \pm 6.27$ & $289.06 \pm 11.70$ & $273.47 \pm 8.17$ \\
\hline 6583 [N II] & $0.97 \pm 0.02$ & $0.85 \pm 0.13$ & $3.40 \pm 0.49$ & $\ldots$ & $\ldots$ & $\ldots$ \\
\hline $6678 \mathrm{He} \mathrm{I}$ & $2.63 \pm 0.05$ & $2.55 \pm 0.15$ & $2.54 \pm 0.44$ & $\ldots$ & $\ldots$ & $\ldots$ \\
\hline 6716 [S II] & $1.89 \pm 0.04$ & $2.58 \pm 0.13$ & $6.89 \pm 0.59$ & $\ldots$ & $\ldots$ & $\ldots$ \\
\hline 6731 [S II] & $1.62 \pm 0.03$ & $1.95 \pm 0.14$ & $4.08 \pm 0.48$ & $\ldots$ & $\ldots$ & $\ldots$ \\
\hline 7065 He I & $4.46 \pm 0.08$ & $2.15 \pm 0.13$ & $2.20 \pm 0.41$ & $\ldots$ & $\ldots$ & $\ldots$ \\
\hline 7136 [Ar III] & $1.57 \pm 0.03$ & $1.47 \pm 0.14$ & $\ldots$ & $\ldots$ & $\ldots$ & $\ldots$ \\
\hline $7281 \mathrm{He}$ I & $0.60 \pm 0.02$ & $0.75 \pm 0.14$ & $\ldots$ & $\ldots$ & $\ldots$ & ... \\
\hline 7320 [O II] & $0.57 \pm 0.02$ & $0.77 \pm 0.18$ & $\ldots$ & $\ldots$ & $\ldots$ & $\ldots$ \\
\hline 7330 [O II] & $0.41 \pm 0.02$ & $0.34 \pm 0.11$ & $\ldots$ & $\ldots$ & $\ldots$ & $\ldots$ \\
\hline$C(\mathrm{H} \beta)$ & 0.135 & 0.090 & 0.485 & 0.180 & 0.115 & 0.040 \\
\hline$E W(\mathrm{H} \beta))^{a}$ & 337 & 305 & 93 & 161 & 53 & 103 \\
\hline$F(\mathrm{H} \beta)^{b}$ & 220.3 & 16.2 & 1.4 & 1.2 & 0.5 & 0.8 \\
\hline$E W(\mathrm{abs})$ & 0.0 & 4.6 & 0.0 & 4.0 & 2.0 & 5.8 \\
\hline
\end{tabular}

${ }^{a}$ in $\AA{ }^{b}$ in units $10^{-16} \mathrm{erg} \mathrm{s}^{-1} \mathrm{~cm}^{-2}$. 
Y. I. Izotov et al.: SBS 0335-052E+W: deep VLT/FORS+UVES spectroscopy, Online Material p 3

Table 3. Extinction-corrected weak emission line fluxes in SBS 0335-052E Nos. $1+2$ (high-resolution FORS observations).

\begin{tabular}{|c|c|c|c|c|c|c|c|}
\hline Line & Flux $^{a}$ & Line & Flux $^{a}$ & Line & Flux ${ }^{a}$ & Line & Flux $^{a}$ \\
\hline $3530 \mathrm{He}$ I & $0.10 \pm 0.10$ & $4169 \mathrm{He}$ I+O II & $0.06 \pm 0.02$ & $4650 \mathrm{O}$ II & $0.02 \pm 0.01$ & $5261[\mathrm{Fe} \mathrm{II}]$ & $0.06 \pm 0.01$ \\
\hline $3554 \mathrm{He} \mathrm{I}$ & $0.22 \pm 0.06$ & $4201 \mathrm{He}$ II & $0.02 \pm 0.01$ & 4702 [Fe III] & $0.11 \pm 0.01$ & $5271[\mathrm{Fe} \mathrm{III}]$ & $0.16 \pm 0.01$ \\
\hline $3587 \mathrm{He} \mathrm{I}$ & $0.31 \pm 0.05$ & 4245 [Fe II] & $0.03 \pm 0.01$ & 4723 [Ne IV] & $0.02 \pm 0.01$ & 5297 O I & $0.04 \pm 0.01$ \\
\hline $3614 \mathrm{He}$ I & $0.22 \pm 0.04$ & 4267 C II & $0.02 \pm 0.01$ & $4734[\mathrm{Fe}$ III] & $0.03 \pm 0.01$ & $5411 \mathrm{He}$ II & $0.10 \pm 0.01$ \\
\hline $3635 \mathrm{He} \mathrm{I}$ & $0.45 \pm 0.06$ & 4287 [Fe II] & $0.15 \pm 0.02$ & $4755[\mathrm{Fe}$ III] & $0.06 \pm 0.01$ & $5513 \mathrm{O} \mathrm{I}$ & $0.03 \pm 0.01$ \\
\hline $3683 \mathrm{H} 20$ & $0.20 \pm 0.02$ & 4320 O II & $0.03 \pm 0.01$ & 4769 [Fe III] & $0.03 \pm 0.01$ & $5577 \mathrm{O} \mathrm{I}$ & $0.01 \pm 0.01$ \\
\hline 3687 H19 & $0.38 \pm 0.03$ & 4348 O II & $0.04 \pm 0.01$ & 4788 N II & $0.05 \pm 0.01$ & 5958 SiII & $0.09 \pm 0.01$ \\
\hline $3692 \mathrm{H} 18$ & $0.66 \pm 0.03$ & 4414 [Fe II $]$ & $0.15 \pm 0.02$ & 4815 [Fe II]+SII & $0.07 \pm 0.01$ & 5979 SiII & $0.07 \pm 0.01$ \\
\hline 3697 H17 & $1.00 \pm 0.04$ & $4438 \mathrm{He} \mathrm{I}$ & $0.09 \pm 0.02$ & $4881[\mathrm{Fe}$ III] & $0.12 \pm 0.02$ & 6046 O I & $0.06 \pm 0.01$ \\
\hline 3704 H16 & $1.82 \pm 0.04$ & 4452 [Fe II $]$ & $0.06 \pm 0.02$ & $4890[\mathrm{Fe} \mathrm{II}]$ & $0.09 \pm 0.02$ & 6347 SiII & $0.11 \pm 0.01$ \\
\hline $3712 \mathrm{H} 15$ & $1.57 \pm 0.04$ & 4510 N III & $0.03 \pm 0.01$ & 4931 [O III] & $0.05 \pm 0.01$ & 6371 SiII & $0.09 \pm 0.01$ \\
\hline $3722 \mathrm{H} 14+[\mathrm{S} \mathrm{III}]$ & $2.31 \pm 0.04$ & 4514 N III & $0.03 \pm 0.01$ & 5041 Si II & $0.20 \pm 0.01$ & 7002 O I & $0.08 \pm 0.02$ \\
\hline 3734 H13 & $2.24 \pm 0.04$ & 4536 N III & $0.01 \pm 0.01$ & $5047 \mathrm{He} \mathrm{I}$ & $0.20 \pm 0.01$ & 7170 [Ar IV] & $0.06 \pm 0.02$ \\
\hline 3856 Si II & $0.07 \pm 0.02$ & 4549 N III & $0.04 \pm 0.02$ & $5056 \mathrm{Si}$ II & $0.08 \pm 0.01$ & 7237 [Ar IV] & $0.04 \pm 0.01$ \\
\hline $3927 \mathrm{He} \mathrm{I}$ & $0.09 \pm 0.02$ & $4563[\mathrm{Mg} \mathrm{I}]$ & $0.03 \pm 0.01$ & $5112[\mathrm{Fe}$ II $]$ & $0.04 \pm 0.01$ & 7263 [Ar IV] & $0.05 \pm 0.01$ \\
\hline $4009 \mathrm{He} \mathrm{I}$ & $0.18 \pm 0.02$ & 4571 Mg I & $0.05 \pm 0.02$ & 5146 [Fe VI] & $0.03 \pm 0.01$ & & \\
\hline $4121 \mathrm{He} \mathrm{I}$ & $0.26 \pm 0.02$ & 4624 N II & $0.02 \pm 0.01$ & $5159[\mathrm{Fe} \mathrm{II}]+[\mathrm{Fe}$ VII $]$ & $0.08 \pm 0.01$ & & \\
\hline $4143 \mathrm{He} \mathrm{I}$ & $0.27 \pm 0.02$ & 4634 N III & $0.01 \pm 0.01$ & $5176[\mathrm{Fe} \mathrm{VI}]$ & $0.04 \pm 0.01$ & & \\
\hline 4154 O II & $0.06 \pm 0.02$ & 4642 N III & $0.03 \pm 0.01$ & 5191 [Ar III] & $0.04 \pm 0.01$ & & \\
\hline
\end{tabular}

${ }^{a} I(\mathrm{H} \beta)=100$. 
Y. I. Izotov et al.: SBS 0335-052E+W: deep VLT/FORS+UVES spectroscopy, Online Material p 4

Table 4. Extinction-corrected emission line fluxes (UVES observations).

\begin{tabular}{|c|c|c|c|c|c|c|c|}
\hline \multirow[b]{2}{*}{ Line } & \multicolumn{6}{|c|}{$0335-052 \mathrm{E}$} & \multirow[b]{2}{*}{$0335-052 \mathbf{W}^{b}$} \\
\hline & Nos. $1+2^{a}$ & Nos. $1+2^{b}$ & Nos. $4+5^{b}$ & Nos. $4+5^{c}$ & Nos. $(1+2)+(4+5)^{b}$ & No. $7^{c}$ & \\
\hline $3346[\mathrm{Ne} \mathrm{V}]$ & & 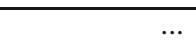 & & 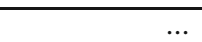 & $0.24 \pm 0.00$ & $\ldots$ & \\
\hline $3425[\mathrm{Ne} \mathrm{v}]$ & $0.97 \pm 0.17$ & & $1.74 \pm 0.03$ & & $0.57 \pm 0.01$ & & \\
\hline 3727 [O II] & $24.84 \pm 0.39$ & $27.22 \pm 0.43$ & $24.03 \pm 0.44$ & $25.26 \pm 0.54$ & $26.19 \pm 0.41$ & $18.79 \pm 0.30$ & $52.06 \pm 0.89$ \\
\hline $3750 \mathrm{H} 12$ & $4.14 \pm 0.08$ & $4.17 \pm 0.08$ & $2.75 \pm 0.15$ & $4.57 \pm 0.31$ & $3.47 \pm 0.09$ & $8.32 \pm 1.27$ & $5.60 \pm 0.27$ \\
\hline 3771 H11 & $5.08 \pm 0.09$ & $4.81 \pm 0.09$ & $3.64 \pm 0.17$ & $5.01 \pm 0.28$ & $4.23 \pm 0.09$ & $5.73 \pm 3.23$ & $6.22 \pm 0.26$ \\
\hline 3797 H10 & $6.41 \pm 0.11$ & $6.21 \pm 0.11$ & $4.79 \pm 0.18$ & $6.68 \pm 0.31$ & $5.51 \pm 0.11$ & $8.82 \pm 1.47$ & $7.53 \pm 0.27$ \\
\hline $3820 \mathrm{He} \mathrm{I}$ & $0.93 \pm 0.03$ & $0.92 \pm 0.03$ & & & $0.83 \pm 0.05$ & & \\
\hline 3835 H9 & $9.30 \pm 0.15$ & $8.28 \pm 0.14$ & $6.51 \pm 0.19$ & $9.30 \pm 0.32$ & $7.61 \pm 0.13$ & $10.96 \pm 1.21$ & $9.20 \pm 0.26$ \\
\hline 3869 [Ne III] & $25.02 \pm 0.38$ & $23.41 \pm 0.36$ & $21.75 \pm 0.37$ & $25.21 \pm 0.46$ & $23.37 \pm 0.36$ & $12.24 \pm 0.59$ & $9.19 \pm 0.20$ \\
\hline $3889 \mathrm{He} \mathrm{I}+\mathrm{H} 8$ & $18.24 \pm 0.28$ & $17.04 \pm 0.27$ & $16.63 \pm 0.34$ & $17.80 \pm 0.42$ & $16.46 \pm 0.27$ & $16.36 \pm 0.94$ & $20.05 \pm 0.42$ \\
\hline 3968 [Ne III] + H7 & & $\ldots$ & $\ldots$ & $\ldots$ & & $7.40 \pm 1.08$ & \\
\hline $4026 \mathrm{He} \mathrm{I}$ & $1.70 \pm 0.04$ & $1.63 \pm 0.04$ & $1.34 \pm 0.11$ & $1.64 \pm 0.17$ & $1.67 \pm 0.06$ & $\ldots$ & $1.31 \pm 0.12$ \\
\hline 4069 [S II] & $0.36 \pm 0.02$ & $0.31 \pm 0.02$ & $\ldots$ & $\ldots$ & $0.30 \pm 0.03$ & $\ldots$ & \\
\hline 4076 [S II] & $0.13 \pm 0.01$ & & & $\ldots$ & & $\ldots$ & \\
\hline $4101 \mathrm{H} \delta$ & $27.92 \pm 0.42$ & $26.22 \pm 0.39$ & $23.67 \pm 0.40$ & $28.84 \pm 0.53$ & $25.64 \pm 0.39$ & $24.88 \pm 0.92$ & $26.59 \pm 0.46$ \\
\hline $4227[\mathrm{Fe} \mathrm{V}]$ & $0.81 \pm 0.06$ & $1.34 \pm 0.11$ & & & $0.76 \pm 0.12$ & & \\
\hline $4340 \mathrm{H} \gamma$ & $48.02 \pm 0.70$ & $47.97 \pm 0.70$ & $48.77 \pm 0.73$ & $49.42 \pm 0.77$ & $48.31 \pm 0.70$ & $46.87 \pm 1.00$ & $47.41 \pm 0.73$ \\
\hline 4363 [O III] & $10.84 \pm 0.16$ & $10.92 \pm 0.17$ & $11.22 \pm 0.23$ & $12.49 \pm 0.28$ & $11.11 \pm 0.18$ & $7.55 \pm 0.65$ & $3.92 \pm 0.15$ \\
\hline $4388 \mathrm{He} \mathrm{I}$ & $0.41 \pm 0.02$ & $0.51 \pm 0.02$ & $\ldots$ & & $0.44 \pm 0.02$ & $\ldots$ & $\ldots$ \\
\hline $4471 \mathrm{He}$ I & $3.58 \pm 0.06$ & $\ldots$ & $\ldots$ & $3.43 \pm 0.12$ & $\ldots$ & $\ldots$ & ... \\
\hline 4658 [Fe III] & $0.34 \pm 0.02$ & $\ldots$ & $\ldots$ & $\ldots$ & $\ldots$ & $\ldots$ & ... \\
\hline $4686 \mathrm{He}$ II & $2.78 \pm 0.05$ & ... & $\ldots$ & $4.36 \pm 0.17$ & $\ldots$ & $\ldots$ & ... \\
\hline 4711 [Ar IV] + He I & & $\cdots$ & $\cdots$ & & & $\ldots$ & $\cdots$ \\
\hline 4740 [Ar IV] & $0.93 \pm 0.03$ & $0.90 \pm 0.01$ & $0.84 \pm 0.03$ & $1.12 \pm 0.05$ & $0.85 \pm 0.02$ & $\ldots$ & \\
\hline $4861 \mathrm{H} \beta$ & $100.00 \pm 1.43$ & $100.00 \pm 1.43$ & $100.00 \pm 1.43$ & $100.00 \pm 1.45$ & $100.00 \pm 1.42$ & $100.00 \pm 1.56$ & $100.00 \pm 1.46$ \\
\hline $4921 \mathrm{He} \mathrm{I}$ & $0.81 \pm 0.03$ & $0.93 \pm 0.02$ & $0.71 \pm 0.02$ & $0.94 \pm 0.06$ & $0.85 \pm 0.02$ & & $0.97 \pm 0.04$ \\
\hline 4959 [O III] & $101.66 \pm 1.45$ & $103.51 \pm 1.48$ & $110.60 \pm 1.58$ & $111.20 \pm 1.61$ & $107.84 \pm 1.53$ & $82.38 \pm 1.32$ & $45.84 \pm 0.68$ \\
\hline 4988 [Fe III] & & & & & & & \\
\hline 5007 [O III] & $311.02 \pm 4.44$ & $309.37 \pm 4.41$ & $337.19 \pm 4.81$ & $332.42 \pm 4.79$ & $326.75 \pm 4.65$ & $247.58 \pm 3.81$ & $137.25 \pm 2.00$ \\
\hline $5016 \mathrm{He} \mathrm{I}$ & $1.86 \pm 0.04$ & $1.77 \pm 0.03$ & $1.73 \pm 0.03$ & $1.71 \pm 0.06$ & $1.70 \pm 0.03$ & $1.79 \pm 0.13$ & $1.96 \pm 0.04$ \\
\hline 5199 [N I] & & & $\ldots$ & $\ldots$ & $\ldots$ & $\ldots$ & $\ldots$ \\
\hline $5518[\mathrm{Cl} \mathrm{III}]$ & $0.13 \pm 0.03$ & $0.11 \pm 0.01$ & $\ldots$ & $\ldots$ & $0.12 \pm 0.01$ & $\ldots$ & ... \\
\hline 5538 [Cl III] & $0.05 \pm 0.02$ & $0.06 \pm 0.00$ & $\ldots$ & $\ldots$ & $0.07 \pm 0.01$ & $\ldots$ & $\ldots$ \\
\hline 575 & & & $\ldots$ & $\ldots$ & $\ldots$ & $\ldots$ & \\
\hline $5876 \mathrm{He}$ I & $9.22 \pm 0.15$ & $11.70 \pm 0.18$ & $8.54 \pm 0.13$ & $9.26 \pm 0.17$ & $10.74 \pm 0.16$ & $9.78 \pm 0.31$ & $8.88 \pm 0.15$ \\
\hline 6300 [O I] & $0.67 \pm 0.03$ & $0.86 \pm 0.02$ & $0.60 \pm 0.02$ & $0.75 \pm 0.04$ & $0.80 \pm 0.02$ & $0.96 \pm 0.11$ & $1.23 \pm 0.04$ \\
\hline 6312 [S III] & $0.55 \pm 0.03$ & $0.67 \pm 0.02$ & $0.56 \pm 0.02$ & $0.64 \pm 0.06$ & $0.65 \pm 0.02$ & $\ldots$ & $0.74 \pm 0.05$ \\
\hline 6364 [O I] & $0.23 \pm 0.02$ & $0.29 \pm 0.01$ & $0.22 \pm 0.02$ & $0.21 \pm 0.03$ & $0.27 \pm 0.01$ & $\ldots$ & $0.36 \pm 0.03$ \\
\hline 6548 [N II] & $0.26 \pm 0.02$ & $0.42 \pm 0.01$ & $0.27 \pm 0.01$ & $0.28 \pm 0.02$ & $0.36 \pm 0.01$ & & $0.60 \pm 0.02$ \\
\hline $6563 \mathrm{H} \alpha$ & $273.64 \pm 4.24$ & $272.68 \pm 4.22$ & $274.28 \pm 4.24$ & $272.73 \pm 4.25$ & $273.10 \pm 4.21$ & $274.73 \pm 4.52$ & $275.55 \pm 4.34$ \\
\hline 6583 [N II] & $0.94 \pm 0.04$ & $1.19 \pm 0.02$ & $0.79 \pm 0.03$ & $0.86 \pm 0.05$ & $1.08 \pm 0.03$ & $0.77 \pm 0.14$ & $1.91 \pm 0.06$ \\
\hline $6678 \mathrm{He} \mathrm{I}$ & $62 \pm 0.05$ & $3.04 \pm 0.05$ & $2.49 \pm 0.05$ & $2.56 \pm 0.05$ & $2.97 \pm 0.05$ & $2.58 \pm 0.09$ & $2.41 \pm 0.06$ \\
\hline 6716 [S II] & $1.89 \pm 0.05$ & $2.01 \pm 0.03$ & $1.82 \pm 0.04$ & $1.62 \pm 0.04$ & $1.98 \pm 0.03$ & $2.10 \pm 0.08$ & $4.97 \pm 0.10$ \\
\hline 6731 [S II] & $1.34 \pm 0.05$ & $\ldots$ & $\ldots$ & $1.32 \pm 0.04$ & $\ldots$ & $1.41 \pm 0.07$ & 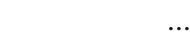 \\
\hline $7065 \mathrm{He}$ I & $17 \pm 0.07$ & $\ldots$ & $\ldots$ & $2.75 \pm 0.06$ & $\ldots$ & $2.02 \pm 0.10$ & ... \\
\hline 7136 [Ar III] & $88 \pm 0.03$ & $\ldots$ & $\ldots$ & $1.45 \pm 0.04$ & $\ldots$ & $1.23 \pm 0.12$ & $\ldots$ \\
\hline $7281 \mathrm{He} \mathrm{I}$ & $0.69 \pm 0.02$ & $\ldots$ & $\ldots$ & $0.57 \pm 0.03$ & $\ldots$ & $\ldots$ & $\ldots$ \\
\hline 7320 [O II] & $0.64 \pm 0.02$ & $\ldots$ & $\ldots$ & $0.38 \pm 0.03$ & $\ldots$ & $\ldots$ & $\ldots$ \\
\hline 7330 [O II] & $0.54 \pm 0.01$ & $\ldots$ & $\ldots$ & $0.38 \pm 0.03$ & $\ldots$ & $\ldots$ & $\ldots$ \\
\hline 9069 [S III] & $4.63 \pm 0.14$ & $\ldots$ & $\ldots$ & $2.80 \pm 0.18$ & $\ldots$ & $2.34 \pm 0.06$ & \\
\hline$C(\mathrm{H} \beta)$ & 0.140 & 0.010 & 0.140 & 0.140 & 0.010 & 0.050 & 0.055 \\
\hline$E W(\mathrm{H} \beta))^{d}$ & 201 & 332 & 104 & 108 & 182 & 280 & 122 \\
\hline$F(\mathrm{H} \beta)^{e}$ & 747.7 & 368.4 & 153.3 & 256.7 & 499.6 & 39.2 & 14.5 \\
\hline$E W(\mathrm{abs})$ & 0.8 & 2.2 & 0.3 & 0.2 & 0.7 & 5.3 & 2.5 \\
\hline
\end{tabular}

${ }^{a}$ ESO program 71.B-0055(A); ${ }^{b}$ ESO program 70.B-0717(A); ${ }^{c}$ ESO program 68.B-0310(A); ${ }^{d}$ in $\AA ;{ }^{e}$ in units $10^{-16} \mathrm{erg} \mathrm{s}^{-1} \mathrm{~cm}^{-2}$. 
Table 5. Ionic and total element abundances (low-resolution FORS observations).

\begin{tabular}{|c|c|c|}
\hline \multirow[b]{2}{*}{ Property } & \multicolumn{2}{|c|}{ "0335-052E } \\
\hline & Nos. $1+2$ & No. 7 \\
\hline$T_{\mathrm{e}}(\mathrm{O}$ III $), \mathrm{K}$ & $20549 \pm 264$ & $20243 \pm 1058$ \\
\hline$T_{\mathrm{e}}(\mathrm{O}$ II $), \mathrm{K}$ & $16356 \pm 185$ & $16320 \pm 753$ \\
\hline$T_{\mathrm{e}}(\mathrm{S}$ III $), \mathrm{K}$ & $18755 \pm 219$ & $18502 \pm 878$ \\
\hline$N_{\mathrm{e}}([\mathrm{S} \mathrm{II}]), \mathrm{cm}^{-3}$ & $541 \pm 97$ & $564 \pm 525$ \\
\hline $\mathrm{O}^{+} / \mathrm{H}^{+},\left(\times 10^{5}\right)$ & $0.174 \pm 0.006$ & $0.192 \pm 0.030$ \\
\hline $\mathrm{O}^{2+} / \mathrm{H}^{+},\left(\times 10^{5}\right)$ & $1.722 \pm 0.053$ & $1.116 \pm 0.132$ \\
\hline $\mathrm{O}^{3+} / \mathrm{H}^{+},\left(\times 10^{6}\right)$ & $0.220 \pm 0.013$ & $\ldots$ \\
\hline $\mathrm{O} / \mathrm{H},\left(\times 10^{5}\right)$ & $1.918 \pm 0.053$ & $1.308 \pm 0.135$ \\
\hline $12+\log \mathrm{O} / \mathrm{H}$ & $7.28 \pm 0.01$ & $7.12 \pm 0.04$ \\
\hline $\mathrm{Ne}^{2+} / \mathrm{H}^{+},\left(\times 10^{6}\right)$ & $2.912 \pm 0.092$ & $2.153 \pm 0.289$ \\
\hline$I C F(\mathrm{Ne})$ & 1.03 & 1.05 \\
\hline $\mathrm{Ne} / \mathrm{H},\left(\times 10^{6}\right)$ & $3.011 \pm 0.102$ & $2.255 \pm 0.339$ \\
\hline $\log \mathrm{Ne} / \mathrm{O}$ & $-0.80 \pm 0.02$ & $-0.76 \pm 0.08$ \\
\hline $\mathrm{N}^{+} / \mathrm{H}^{+},\left(\times 10^{7}\right)$ & $0.563 \pm 0.021$ & $0.633 \pm 0.158$ \\
\hline$I C F(\mathrm{~N})$ & 10.21 & 6.52 \\
\hline $\mathrm{N} / \mathrm{H},\left(\times 10^{7}\right)$ & $5.818 \pm 0.234$ & $4.126 \pm 1.077$ \\
\hline $\log \mathrm{N} / \mathrm{O}$ & $-1.52 \pm 0.02$ & $-1.50 \pm 0.12$ \\
\hline $\mathrm{S}^{+} / \mathrm{H}^{+},\left(\times 10^{7}\right)$ & $0.298 \pm 0.008$ & $0.446 \pm 0.056$ \\
\hline $\mathrm{S}^{2+} / \mathrm{H}^{+},\left(\times 10^{7}\right)$ & $1.680 \pm 0.111$ & $\ldots$ \\
\hline $\operatorname{ICF}(\mathrm{S})$ & 2.22 & $\ldots$ \\
\hline $\mathrm{S} / \mathrm{H},\left(\times 10^{7}\right)$ & $4.440 \pm 0.248$ & $\ldots$ \\
\hline $\log \mathrm{S} / \mathrm{O}$ & $-1.64 \pm 0.03$ & $\ldots$ \\
\hline $\mathrm{Cl}^{2+} / \mathrm{H}^{+},\left(\times 10^{8}\right)$ & $0.348 \pm 0.075$ & $\ldots$ \\
\hline $\operatorname{ICF}(\mathrm{Cl})$ & 1.38 & $\ldots$ \\
\hline $\mathrm{Cl} / \mathrm{H},\left(\times 10^{8}\right)$ & $0.480 \pm 0.103$ & $\ldots$ \\
\hline $\log \mathrm{Cl} / \mathrm{O}$ & $-3.60 \pm 0.09$ & $\ldots$ \\
\hline $\mathrm{Ar}^{2+} / \mathrm{H}^{+},\left(\times 10^{7}\right)$ & $0.426 \pm 0.014$ & $0.308 \pm 0.069$ \\
\hline $\mathrm{Ar}^{3+} / \mathrm{H}^{+},\left(\times 10^{7}\right)$ & $0.413 \pm 0.028$ & 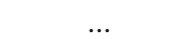 \\
\hline $\operatorname{ICF}(\mathrm{Ar})$ & 2.14 & 1.53 \\
\hline $\mathrm{Ar} / \mathrm{H},\left(\times 10^{7}\right)$ & $0.912 \pm 0.068$ & $0.471 \pm 0.106$ \\
\hline $\log \mathrm{Ar} / \mathrm{O}$ & $-2.32 \pm 0.03$ & $-2.44 \pm 0.11$ \\
\hline $\mathrm{Fe}^{2+} / \mathrm{H}^{+},\left(\times 10^{7}\right)(4658)$ & $0.572 \pm 0.073$ & $\ldots$ \\
\hline$I C F(\mathrm{Fe})$ & 15.25 & $\ldots$ \\
\hline $\mathrm{Fe} / \mathrm{H},\left(\times 10^{7}\right)(4658)$ & $8.729 \pm 1.119$ & $\ldots$ \\
\hline $\log \mathrm{Fe} / \mathrm{O}(4658)$ & $-1.34 \pm 0.06$ & $\ldots$ \\
\hline $\mathrm{Fe}^{2+} / \mathrm{H}^{+},\left(\times 10^{7}\right)(4988)$ & $0.724 \pm 0.073$ & $\ldots$ \\
\hline$I C F(\mathrm{Fe})$ & 15.25 & $\ldots$ \\
\hline $\mathrm{Fe} / \mathrm{H},\left(\times 10^{7}\right)(4988)$ & $11.050 \pm 1.118$ & $\ldots$ \\
\hline $\log \mathrm{Fe} / \mathrm{O}(4988)$ & $-1.24 \pm 0.05$ & $\ldots$ \\
\hline
\end{tabular}


Y. I. Izotov et al.: SBS 0335-052E+W: deep VLT/FORS+UVES spectroscopy, Online Material p 6

Table 6. Ionic and total element abundances (high-resolution FORS observations).

\begin{tabular}{|c|c|c|c|c|c|c|}
\hline \multirow[b]{2}{*}{ Property } & \multicolumn{2}{|c|}{$0335-052 \mathrm{E}$} & \multicolumn{4}{|c|}{$0335-052 \mathrm{~W}$} \\
\hline & Nos. $1+2$ & No. 7 & No. 1 & No. 2 & No. 3 & No. 4 \\
\hline$T_{\mathrm{e}}(\mathrm{O}$ III $), \mathrm{K}$ & $21314 \pm 257$ & $19281 \pm 352$ & $17188 \pm 1557$ & $20273 \pm 1771$ & $20000 \pm 1048$ & $20059 \pm 3899$ \\
\hline$T_{\mathrm{e}}(\mathrm{O} \mathrm{II}), \mathrm{K}$ & $16407 \pm 171$ & $16145 \pm 263$ & $15452 \pm 1268$ & $16323 \pm 1259$ & $16284 \pm 1329$ & $16293 \pm 2804$ \\
\hline$T_{\mathrm{e}}(\mathrm{S}$ III $), \mathrm{K}$ & $19390 \pm 213$ & $17703 \pm 292$ & $15966 \pm 1293$ & $18526 \pm 1470$ & $18300 \pm 870$ & $18349 \pm 3236$ \\
\hline$N_{\mathrm{e}}([\mathrm{S} \mathrm{II}]), \mathrm{cm}^{-3}$ & $316 \pm 54$ & $100 \pm 118$ & 100 & 100 & 100 & 100 \\
\hline $\mathrm{O}^{+} / \mathrm{H}^{+},\left(\times 10^{5}\right)$ & $0.156 \pm 0.004$ & $0.183 \pm 0.008$ & $0.623 \pm 0.131$ & $0.212 \pm 0.042$ & $0.561 \pm 0.124$ & $0.297 \pm 0.127$ \\
\hline $\mathrm{O}^{2+} / \mathrm{H}^{+},\left(\times 10^{5}\right)^{a}$ & $1.526 \pm 0.044$ & $1.119 \pm 0.052$ & $1.032 \pm 0.226$ & $0.822 \pm 0.162$ & $0.403 \pm 0.052$ & $0.421 \pm 0.185$ \\
\hline $\mathrm{O}^{2+} / \mathrm{H}^{+},\left(\times 10^{5}\right)^{b}$ & $1.500 \pm 0.750$ & $\ldots$ & $\ldots$ & $\ldots$ & $\ldots$ & $\ldots$ \\
\hline $\mathrm{O}^{3+} / \mathrm{H}^{+},\left(\times 10^{6}\right)$ & $0.230 \pm 0.009$ & $0.204 \pm 0.025$ & $\ldots$ & $\ldots$ & $\ldots$ & $\ldots$ \\
\hline $\mathrm{O} / \mathrm{H},\left(\times 10^{5}\right)$ & $1.705 \pm 0.044$ & $1.403 \pm 0.053$ & $1.654 \pm 0.262$ & $1.034 \pm 0.167$ & $0.965 \pm 0.134$ & $0.718 \pm 0.224$ \\
\hline $12+\log \mathrm{O} / \mathrm{H}$ & $7.23 \pm 0.01$ & $7.15 \pm 0.02$ & $7.22 \pm 0.07$ & $7.01 \pm 0.07$ & $6.98 \pm 0.06$ & $6.86 \pm 0.14$ \\
\hline $\mathrm{Ne}^{2+} / \mathrm{H}^{+},\left(\times 10^{6}\right)$ & $2.960 \pm 0.084$ & $2.189 \pm 0.099$ & $2.078 \pm 0.480$ & $1.526 \pm 0.306$ & $\ldots$ & $\ldots$ \\
\hline$I C F(\mathrm{Ne})$ & 1.03 & 1.05 & 1.14 & 1.07 & $\ldots$ & $\ldots$ \\
\hline $\mathrm{Ne} / \mathrm{H},\left(\times 10^{6}\right)$ & $3.064 \pm 0.094$ & $2.291 \pm 0.116$ & $2.375 \pm 0.077$ & $1.629 \pm 0.384$ & $\ldots$ & $\ldots$ \\
\hline $\log \mathrm{Ne} / \mathrm{O}$ & $-0.74 \pm 0.02$ & $-0.79 \pm 0.03$ & $-0.84 \pm 0.16$ & $-0.80 \pm 0.12$ & $\ldots$ & $\ldots$ \\
\hline $\mathrm{N}^{+} / \mathrm{H}^{+},\left(\times 10^{7}\right)$ & $0.600 \pm 0.015$ & $0.543 \pm 0.067$ & $2.364 \pm 0.426$ & $\ldots$ & $\ldots$ & $\ldots$ \\
\hline$I C F(\mathrm{~N})$ & 10.21 & 7.29 & 2.68 & $\ldots$ & $\ldots$ & $\ldots$ \\
\hline $\mathrm{N} / \mathrm{H},\left(\times 10^{7}\right)$ & $6.126 \pm 0.163$ & $3.958 \pm 0.514$ & $6.339 \pm 1.132$ & $\ldots$ & $\ldots$ & $\ldots$ \\
\hline $\log \mathrm{N} / \mathrm{O}$ & $-1.44 \pm 0.02$ & $-1.55 \pm 0.06$ & $-1.42 \pm 0.10$ & $\ldots$ & $\ldots$ & $\ldots$ \\
\hline $\mathrm{S}^{+} / \mathrm{H}^{+},\left(\times 10^{7}\right)$ & $0.299 \pm 0.006$ & $0.387 \pm 0.019$ & $\cdots$ & $\ldots$ & $\ldots$ & $\ldots$ \\
\hline $\mathrm{S}^{2+} / \mathrm{H}^{+},\left(\times 10^{7}\right)$ & $1.486 \pm 0.056$ & $1.621 \pm 0.394$ & $\ldots$ & $\ldots$ & $\ldots$ & $\ldots$ \\
\hline$I C F(\mathrm{~S})$ & 2.20 & 1.72 & $\ldots$ & $\ldots$ & $\ldots$ & $\ldots$ \\
\hline $\mathrm{S} / \mathrm{H},\left(\times 10^{7}\right)$ & $3.937 \pm 0.124$ & $3.464 \pm 0.681$ & $\ldots$ & $\ldots$ & $\ldots$ & $\ldots$ \\
\hline $\log \mathrm{S} / \mathrm{O}$ & $-1.64 \pm 0.02$ & $-1.61 \pm 0.08$ & $\ldots$ & $\ldots$ & $\ldots$ & $\ldots$ \\
\hline $\mathrm{Cl}^{2+} / \mathrm{H}^{+},\left(\times 10^{8}\right)$ & $0.249 \pm 0.035$ & $\cdots$ & $\cdots$ & $\ldots$ & $\ldots$ & $\ldots$ \\
\hline $\operatorname{ICF}(\mathrm{Cl})$ & 1.38 & $\ldots$ & $\ldots$ & $\ldots$ & $\ldots$ & $\ldots$ \\
\hline $\mathrm{Cl} / \mathrm{H},\left(\times 10^{8}\right)$ & $0.343 \pm 0.048$ & $\ldots$ & $\ldots$ & $\ldots$ & $\ldots$ & $\ldots$ \\
\hline $\log \mathrm{Cl} / \mathrm{O}$ & $-3.70 \pm 0.06$ & $\ldots$ & $\ldots$ & $\ldots$ & $\ldots$ & $\ldots$ \\
\hline $\mathrm{Ar}^{2+} / \mathrm{H}^{+},\left(\times 10^{7}\right)$ & $0.417 \pm 0.009$ & $0.448 \pm 0.043$ & $\cdots$ & $\ldots$ & $\ldots$ & $\ldots$ \\
\hline $\mathrm{Ar}^{3+} / \mathrm{H}^{+},\left(\times 10^{7}\right)$ & $0.412 \pm 0.012$ & $0.244 \pm 0.069$ & $\cdots$ & $\ldots$ & $\ldots$ & $\ldots$ \\
\hline$I C F(\mathrm{Ar})$ & 2.12 & 1.65 & $\ldots$ & $\ldots$ & $\ldots$ & $\ldots$ \\
\hline $\mathrm{Ar} / \mathrm{H},\left(\times 10^{7}\right)$ & $0.885 \pm 0.033$ & $0.739 \pm 0.134$ & $\ldots$ & $\ldots$ & $\ldots$ & $\ldots$ \\
\hline $\log \mathrm{Ar} / \mathrm{O}$ & $-2.28 \pm 0.02$ & $-2.28 \pm 0.08$ & $\ldots$ & $\ldots$ & $\ldots$ & $\ldots$ \\
\hline $\mathrm{Fe}^{2+} / \mathrm{H}^{+},\left(\times 10^{7}\right)(4658)$ & $0.498 \pm 0.029$ & $0.644 \pm 0.150$ & $\ldots$ & $\ldots$ & $\ldots$ & $\ldots$ \\
\hline$I C F(\mathrm{Fe})$ & 15.08 & 10.53 & $\ldots$ & $\ldots$ & $\ldots$ & $\ldots$ \\
\hline $\mathrm{Fe} / \mathrm{H},\left(\times 10^{7}\right)(4658)$ & $7.506 \pm 0.434$ & $6.787 \pm 1.585$ & $\ldots$ & $\ldots$ & $\ldots$ & $\ldots$ \\
\hline $\log \mathrm{Fe} / \mathrm{O}(4658)$ & $-1.36 \pm 0.03$ & $-1.32 \pm 0.10$ & $\ldots$ & $\ldots$ & $\ldots$ & $\ldots$ \\
\hline $\mathrm{Fe}^{2+} / \mathrm{H}^{+},\left(\times 10^{7}\right)(4988)$ & $0.589 \pm 0.026$ & $0.961 \pm 0.131$ & $\ldots$ & $\ldots$ & $\ldots$ & $\ldots$ \\
\hline$I C F(\mathrm{Fe})$ & 15.08 & 10.53 & $\ldots$ & $\ldots$ & $\ldots$ & $\ldots$ \\
\hline $\mathrm{Fe} / \mathrm{H},\left(\times 10^{7}\right)(4988)$ & $8.875 \pm 0.386$ & $10.120 \pm 1.380$ & $\cdots$ & $\ldots$ & $\ldots$ & $\ldots$ \\
\hline $\log \mathrm{Fe} / \mathrm{O}(4988)$ & $-1.28 \pm 0.02$ & $-1.14 \pm 0.06$ & $\ldots$ & $\ldots$ & $\ldots$ & $\ldots$ \\
\hline
\end{tabular}

${ }^{a}$ Derived from the fluxes of the [O III] $\lambda 4959,5007$ emission lines ${ }^{b}$ derived from the flux of the O II $\lambda 4650$ recombination emission line. 
Table 7. Ionic and total element abundances (UVES observations).

\begin{tabular}{|c|c|c|c|c|c|c|c|}
\hline \multirow[b]{2}{*}{ Property } & \multicolumn{6}{|c|}{$0335-052 \mathrm{E}$} & \multirow[b]{2}{*}{$0335-052 \mathrm{~W}^{b}$} \\
\hline & Nos. $1+2^{a}$ & Nos. $1+2^{b}$ & Nos. $4+5^{b}$ & Nos. $4+5^{c}$ & Nos. $(1+2)+(4+5)^{b}$ & No. $7^{c}$ & \\
\hline$T_{\mathrm{e}}(\mathrm{O}$ III $), \mathrm{K}$ & $20487 \pm 240$ & $20585 \pm 246$ & $19915 \pm 283$ & $21386 \pm 349$ & $20158 \pm 239$ & $18892 \pm 945$ & $18196 \pm 390$ \\
\hline$T_{\mathrm{e}}(\mathrm{O}$ II $), \mathrm{K}$ & $16349 \pm 169$ & $16360 \pm 172$ & $16270 \pm 205$ & $16409 \pm 232$ & $16308 \pm 171$ & $16049 \pm 719$ & $15839 \pm 306$ \\
\hline$T_{\mathrm{e}}(\mathrm{S}$ III $), \mathrm{K}$ & $18704 \pm 199$ & $18786 \pm 204$ & $18229 \pm 235$ & $19450 \pm 290$ & $18431 \pm 199$ & $17381 \pm 784$ & $16802 \pm 324$ \\
\hline$N_{\mathrm{e}}([\mathrm{S} \mathrm{II}]), \mathrm{cm}^{-3}$ & 100 & 100 & 100 & $218 \pm 65$ & 100 & 100 & 100 \\
\hline $\mathrm{O}^{+} / \mathrm{H}^{+},\left(\times 10^{5}\right)$ & $0.174 \pm 0.005$ & $0.190 \pm 0.006$ & $0.171 \pm 0.006$ & $0.179 \pm 0.007$ & $0.185 \pm 0.006$ & $0.139 \pm 0.015$ & $0.400 \pm 0.020$ \\
\hline $\mathrm{O}^{2+} / \mathrm{H}^{+},\left(\times 10^{5}\right)$ & $1.684 \pm 0.048$ & $1.668 \pm 0.048$ & $1.936 \pm 0.066$ & $1.661 \pm 0.062$ & $1.832 \pm 0.053$ & $1.593 \pm 0.185$ & $0.959 \pm 0.050$ \\
\hline $\mathrm{O}^{3+} / \mathrm{H}^{+},\left(\times 10^{6}\right)$ & $0.562 \pm 0.021$ & $\ldots$ & $\ldots$ & $0.908 \pm 0.050$ & $\ldots$ & $\ldots$ & .. \\
\hline $\mathrm{O} / \mathrm{H},\left(\times 10^{5}\right)$ & $1.914 \pm 0.048$ & $1.859 \pm 0.049$ & $2.106 \pm 0.066$ & $1.931 \pm 0.063$ & $2.017 \pm 0.053$ & $1.732 \pm 0.186$ & $1.359 \pm 0.054$ \\
\hline $12+\log \mathrm{O} / \mathrm{H}$ & $7.28 \pm 0.01$ & $7.27 \pm 0.01$ & $7.32 \pm 0.01$ & $7.28 \pm 0.01$ & $7.30 \pm 0.01$ & $7.24 \pm 0.05$ & $7.13 \pm 0.02$ \\
\hline $\mathrm{Ne}^{2+} / \mathrm{H}^{+},\left(\times 10^{6}\right)$ & $2.999 \pm 0.085$ & $2.776 \pm 0.080$ & $2.782 \pm 0.096$ & $2.746 \pm 0.102$ & $2.906 \pm 0.084$ & $1.774 \pm 0.212$ & $0.297 \pm 0.127$ \\
\hline$I C F(\mathrm{Ne})$ & 1.04 & 1.03 & 1.03 & 1.04 & 1.03 & 1.03 & 1.03 \\
\hline $\mathrm{Ne} / \mathrm{H},\left(\times 10^{6}\right)$ & $3.117 \pm 0.097$ & $2.871 \pm 0.089$ & $2.862 \pm 0.104$ & $2.870 \pm 0.119$ & $2.998 \pm 0.093$ & $1.824 \pm 0.231$ & $0.297 \pm 0.127$ \\
\hline $\log \mathrm{Ne} / \mathrm{O}$ & $-0.79 \pm 0.02$ & $-0.81 \pm 0.02$ & $-0.87 \pm 0.02$ & $-0.83 \pm 0.02$ & $-0.83 \pm 0.02$ & $-0.98 \pm 0.07$ & $-0.98 \pm 0.07$ \\
\hline $\mathrm{N}^{+} / \mathrm{H}^{+},\left(\times 10^{7}\right)$ & $0.590 \pm 0.022$ & $0.740 \pm 0.017$ & $0.500 \pm 0.018$ & $0.532 \pm 0.027$ & $0.680 \pm 0.017$ & $0.496 \pm 0.080$ & $0.297 \pm 0.127$ \\
\hline$I C F(\mathrm{~N})$ & 10.28 & 9.18 & 11.47 & 10.09 & 10.20 & 11.58 & 11.58 \\
\hline $\mathrm{N} / \mathrm{H},\left(\times 10^{7}\right)$ & $6.059 \pm 0.238$ & $6.795 \pm 0.016$ & $5.735 \pm 0.217$ & $5.373 \pm 0.288$ & $6.933 \pm 0.185$ & $5.751 \pm 1.001$ & $0.297 \pm 0.127$ \\
\hline $\log \mathrm{N} / \mathrm{O}$ & $-1.50 \pm 0.02$ & $-1.44 \pm 0.02$ & $-1.56 \pm 0.02$ & $-1.56 \pm 0.03$ & $-1.46 \pm 0.02$ & $-1.48 \pm 0.09$ & $-1.48 \pm 0.09$ \\
\hline $\mathrm{S}^{+} / \mathrm{H}^{+},\left(\times 10^{7}\right)$ & $0.267 \pm 0.007$ & $0.166 \pm 0.004$ & $0.152 \pm 0.004$ & $0.248 \pm 0.007$ & $0.165 \pm 0.004$ & .. & $0.435 \pm 0.014$ \\
\hline $\mathrm{S}^{2+} / \mathrm{H}^{+},\left(\times 10^{7}\right)$ & $1.521 \pm 0.100$ & $1.829 \pm 0.061$ & $1.657 \pm 0.085$ & $1.601 \pm 0.155$ & $1.857 \pm 0.075$ & $\begin{array}{l}\cdots \\
\cdots\end{array}$ & $2.713 \pm 0.215$ \\
\hline$I C F(\mathrm{~S})$ & 2.22 & 2.03 & 2.41 & 2.19 & 2.20 & $\ldots$ & 1.10 \\
\hline $\mathrm{S} / \mathrm{H},\left(\times 10^{7}\right)$ & $3.963 \pm 0.223$ & $4.060 \pm 0.124$ & $4.368 \pm 0.206$ & $4.043 \pm 0.338$ & $4.457 \pm 0.166$ & $\ldots$ & $3.474 \pm 0.238$ \\
\hline $\log \mathrm{S} / \mathrm{O}$ & $-1.68 \pm 0.03$ & $-1.66 \pm 0.02$ & $-1.68 \pm 0.02$ & $-1.68 \pm 0.04$ & $-1.66 \pm 0.02$ & $\ldots$ & $-1.59 \pm 0.03$ \\
\hline $\mathrm{Cl}^{2+} / \mathrm{H}^{+},\left(\times 10^{8}\right)$ & $0.298 \pm 0.056$ & $0.272 \pm 0.013$ & $\ldots$ & $\ldots$ & $0.317 \pm 0.024$ & $\ldots$ & $\ldots$ \\
\hline$I C F(\mathrm{Cl})$ & 1.38 & 1.32 & $\ldots$ & $\ldots$ & 1.38 & $\ldots$ & $\ldots$ \\
\hline $\mathrm{Cl} / \mathrm{H},\left(\times 10^{8}\right)$ & $0.410 \pm 0.078$ & $0.361 \pm 0.017$ & $\ldots$ & $\ldots$ & $0.436 \pm 0.033$ & $\ldots$ & $\ldots$ \\
\hline $\log \mathrm{Cl} / \mathrm{O}$ & $-3.67 \pm 0.08$ & $-3.71 \pm 0.02$ & $\ldots$ & $\ldots$ & $-3.66 \pm 0.04$ & $\ldots$ & $\ldots$ \\
\hline $\mathrm{Ar}^{2+} / \mathrm{H}^{+},\left(\times 10^{7}\right)$ & $0.525 \pm 0.011$ & $\ldots$ & $\ldots$ & $0.383 \pm 0.012$ & $\ldots$ & $0.385 \pm 0.040$ & $\ldots$ \\
\hline $\mathrm{Ar}^{3+} / \mathrm{H}^{+},\left(\times 10^{7}\right)$ & $0.451 \pm 0.015$ & $\ldots$ & $\ldots$ & $0.495 \pm 0.026$ & $\ldots$ & $\ldots$ & $\ldots$ \\
\hline $\operatorname{ICF}(\mathrm{Ar})$ & 2.13 & $\ldots$ & $\ldots$ & 2.10 & $\ldots$ & 2.35 & $\ldots$ \\
\hline $\mathrm{Ar} / \mathrm{H},\left(\times 10^{7}\right)$ & $1.120 \pm 0.039$ & $\ldots$ & $\ldots$ & $0.806 \pm 0.062$ & $\ldots$ & $0.904 \pm 0.095$ & $\ldots$ \\
\hline $\log \mathrm{Ar} / \mathrm{O}$ & $-2.23 \pm 0.02$ & $\ldots$ & $\ldots$ & $-2.38 \pm 0.04$ & $\ldots$ & $-2.28 \pm 0.06$ & $\ldots$ \\
\hline $\mathrm{Fe}^{2+} / \mathrm{H}^{+},\left(\times 10^{7}\right)(4658)$ & $0.526 \pm 0.026$ & $\ldots$ & $\ldots$ & $\ldots$ & $\ldots$ & $\ldots$ & $\ldots$ \\
\hline$I C F(\mathrm{Fe})$ & 15.18 & $\ldots$ & $\ldots$ & $\ldots$ & $\ldots$ & $\ldots$ & $\ldots$ \\
\hline $\mathrm{Fe} / \mathrm{H},\left(\times 10^{7}\right)(4658)$ & $7.986 \pm 0.397$ & $\ldots$ & $\ldots$ & $\ldots$ & $\ldots$ & $\ldots$ & $\ldots$ \\
\hline $\log \mathrm{Fe} / \mathrm{O}(4658)$ & $-1.38 \pm 0.02$ & $\ldots$ & $\ldots$ & $\ldots$ & $\ldots$ & $\ldots$ & $\ldots$ \\
\hline $\mathrm{Fe}^{2+} / \mathrm{H}^{+},\left(\times 10^{7}\right)(4988)$ & $\ldots$ & $\ldots$ & $\ldots$ & $\ldots$ & $\ldots$ & $\ldots$ & $\ldots$ \\
\hline$I C F(\mathrm{Fe})$ & $\ldots$ & $\ldots$ & $\ldots$ & $\ldots$ & $\ldots$ & $\ldots$ & $\ldots$ \\
\hline $\mathrm{Fe} / \mathrm{H},\left(\times 10^{7}\right)(4988)$ & $\ldots$ & $\ldots$ & $\ldots$ & $\ldots$ & $\ldots$ & $\ldots$ & $\ldots$ \\
\hline $\log \mathrm{Fe} / \mathrm{O}(4988)$ & $\ldots$ & $\ldots$ & $\ldots$ & $\ldots$ & $\ldots$ & $\ldots$ & $\ldots$ \\
\hline
\end{tabular}

${ }^{a}$ ESO program 71.B-0055(A); ${ }^{b}$ ESO program 70.B-0717(A); ${ }^{c}$ ESO program 68.B-0310(A). 
Y. I. Izotov et al.: SBS 0335-052E+W: deep VLT/FORS+UVES spectroscopy, Online Material p 8

Table 8. Helium abundances.

\begin{tabular}{|c|c|c|c|c|c|c|c|}
\hline \multirow[b]{2}{*}{ Property } & \multicolumn{6}{|c|}{$0335-052 \mathrm{E}$} & \multirow[b]{2}{*}{$0335-052 \mathrm{~W}^{b}$} \\
\hline & Nos. $1+2^{a}$ & Nos. $1+2^{b}$ & Nos. $1+2^{c}$ & Nos. $4+5^{c}$ & No. $7^{a}$ & No. $7^{b}$ & \\
\hline \multicolumn{8}{|c|}{ a) Benjamin et al. (2002) emissivities } \\
\hline$\Delta I(\mathrm{H} \alpha) / I(\mathrm{H} \alpha)$ & 0.0004 & 0.0013 & 0.0177 & 0.0007 & 0.0500 & 0.0491 & 0.0490 \\
\hline$T_{\mathrm{e}}\left(\mathrm{He}^{+}\right)$ & 19520 & 20250 & 20410 & 20330 & 19110 & 18970 & \\
\hline$N_{\mathrm{e}}\left(\mathrm{He}^{+}\right)$ & 162 & 132 & 53 & 12 & 12 & 10 & 14 \\
\hline$\tau(\lambda 3889)$ & 4.59 & 5.01 & 5.01 & 1.24 & 0.01 & 0.02 & 0.02 \\
\hline$y^{+}(\lambda 4471)$ & $0.0834 \pm 0.0021$ & $0.0820 \pm 0.0013$ & $0.0827 \pm 0.0013$ & $0.0834 \pm 0.0028$ & $0.0858 \pm 0.0118$ & $0.0812 \pm 0.0030$ & $0.0935 \pm 0.0086$ \\
\hline$y^{+}(\lambda 5876)$ & $852 \pm 0.0014$ & $0839 \pm 0.0013$ & $0.0777 \pm 0.0012$ & $0.0798 \pm 0.0015$ & $0.0816 \pm 0.0$ & $0.0802 \pm 0.0$ & \\
\hline$y^{+}(\lambda 6678)$ & $768 \pm 0.0017$ & $0798 \pm 0.0014$ & $0.0818 \pm 0.0017$ & $0.0792 \pm 0.0$ & $876 \pm 0.0$ & $812 \pm 0.0$ & \\
\hline$y_{w m}^{+}$ & $822 \pm 0.0010$ & $.0821 \pm 0.0008$ & $0.0805 \pm 0.0008$ & $0.0801 \pm 0.0010$ & $0.0826 \pm 0.0031$ & $0.0806 \pm 0.0016$ & 0.0 \\
\hline$y^{2+}(\lambda 4686)$ & $0.0011 \pm 0.0001$ & $0.0012 \pm 0.0000$ & $0.0026 \pm 0.0000$ & $0.0041 \pm 0.0002$ & & $0.0012 \pm 0.0001$ & \\
\hline$I C F$ & 0.9936 & 0.9935 & 0.9933 & 0.9928 & 0.9928 & 0.9929 & \\
\hline$y(\lambda 4471)$ & $.0839 \pm 0.0021$ & $.0827 \pm 0.0013$ & $0.0848 \pm 0.0013$ & $0.0868 \pm 0.00$ & $0.0852 \pm 0.0117$ & $0.0818 \pm 0$ & 0.0085 \\
\hline & & $6 \pm 0.0013$ & $8 \pm 0.0012$ & $0.0833 \pm 0$ & $11 \pm 0$ & $09 \pm$ & 0064 \\
\hline$y(\lambda 667$ & & $5 \pm 0.0014$ & \pm 0.0017 & $27 \pm$ & 92 & $19 \pm$ & 0135 \\
\hline$y_{w m}$ & & $328 \pm 0.0008$ & 0.08 & $0.0835 \pm$ & $20 \pm$ & $12 \pm$ & \\
\hline$Y$ & 30 & $486 \pm 0.0024$ & $0.2481 \pm 0.0024$ & $0.2503 \pm 0$. & $0.2470 \pm$ & $0.2452 \pm$ & 0.2 \\
\hline \multicolumn{8}{|c|}{ b) Porter et al. (2005) emissivities } \\
\hline & & 0.0027 & 0.0322 & 0.0002 & & & \\
\hline & 19520 & 20260 & 20360 & 20330 & 19060 & 18850 & \\
\hline & 197 & 162 & 86 & 10 & 11 & 11 & 11 \\
\hline$\tau(\lambda 3889)$ & 4.52 & 5.00 & 5.01 & 1.44 & 0.02 & 0.02 & 0.04 \\
\hline & $0853 \pm 0.0021$ & $0.0841 \pm 0.0013$ & $0.0849 \pm 0.0013$ & $0.0862 \pm 0.0028$ & $0.0884 \pm 0.0118$ & $0.0835 \pm 0.0030$ & $0.0958 \pm 0.0086$ \\
\hline$y^{+}(\lambda 5876)$ & $860 \pm 0.0014$ & $.0848 \pm 0.0013$ & $0.0792 \pm 0.0012$ & $0.0816 \pm 0.0$ & $0.0835 \pm 0$. & $820 \pm 0$. & $00 \pm 0.0065$ \\
\hline$y^{+}(\lambda 6678)$ & $0771 \pm 0.0017$ & $0800 \pm 0.0014$ & $0.0831 \pm 0.0017$ & $0.0796 \pm 0.0016$ & $0.0882 \pm 0.0$ & $0.0818 \pm 0.0049$ & \\
\hline$y_{w m}^{+}$ & $0.0831 \pm 0.0010$ & $0.0831 \pm 0.0008$ & $0.0821 \pm 0.0008$ & $0.0814 \pm 0.0010$ & $0.0844 \pm 0.0031$ & $0.0824 \pm 0.0016$ & $0.0851 \pm 0.0048$ \\
\hline$y^{2+}(\lambda 4686)$ & $0.0011 \pm 0.0001$ & $0.0012 \pm 0.0000$ & $0.0026 \pm 0.0000$ & $0.0041 \pm 0.0002$ & & $0.0012 \pm 0.0001$ & \\
\hline$I C F$ & 0.9936 & 0.9935 & 0.9933 & 0.9928 & 0.9928 & 0.9929 & \\
\hline$y(\lambda 4471)$ & $0858 \pm 0.0021$ & $0.0848 \pm 0.0013$ & $0.0869 \pm 0.0013$ & $0.0897 \pm 0.0028$ & $0.0877 \pm 0.0117$ & $0.0842 \pm 0.0030$ & $0.0948 \pm 0.0085$ \\
\hline$y(\lambda 5876)$ & $865 \pm 0.0013$ & $0854 \pm 0.0012$ & $0.0812 \pm 0.0012$ & $0.0851 \pm 0.0015$ & $0.0829 \pm 0.0035$ & $0.0827 \pm 0.0020$ & $0.0792 \pm 0.0064$ \\
\hline$y(\lambda 6678)$ & $0776 \pm 0.0017$ & $.0807 \pm 0.0014$ & $0.0851 \pm 0.0017$ & $0.0831 \pm 0.0016$ & $0.0876 \pm 0.0091$ & $0.0825 \pm 0.0049$ & $0.0794 \pm 0.0135$ \\
\hline$y_{w m}$ & $.0836 \pm 0.0009$ & $0.0838 \pm 0.0008$ & $0.0842 \pm 0.0008$ & $0.0849 \pm 0.0010$ & $0.0838 \pm 0.0031$ & $0.0831 \pm 0.0016$ & $0.0842 \pm 0.0048$ \\
\hline$Y$ & $0.2506 \pm 0.0029$ & $0.2510 \pm 0.0023$ & $0.2518 \pm 0.0024$ & $0.2533 \pm 0.0031$ & $0.2509 \pm 0.0096$ & $0.2493 \pm 0.0048$ & $0.2518 \pm 0.0148$ \\
\hline
\end{tabular}

${ }^{a}$ Low-resolution FORS observations; ${ }^{b}$ high-resolution FORS observations; ${ }^{c}$ UVES observations. 